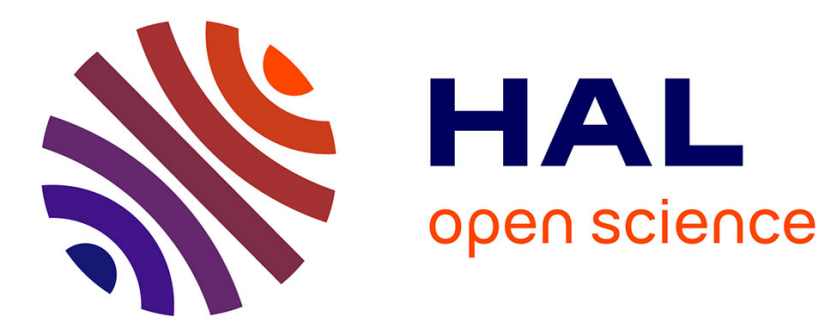

\title{
A two-dimensional version of the Godunov scheme for scalar balance laws \\ Laurent Gosse
}

\section{To cite this version:}

Laurent Gosse. A two-dimensional version of the Godunov scheme for scalar balance laws. 2013. hal-00870221v1

\section{HAL Id: hal-00870221 \\ https://hal.science/hal-00870221v1}

Preprint submitted on 6 Oct 2013 (v1), last revised 21 Nov 2013 (v2)

HAL is a multi-disciplinary open access archive for the deposit and dissemination of scientific research documents, whether they are published or not. The documents may come from teaching and research institutions in France or abroad, or from public or private research centers.
L'archive ouverte pluridisciplinaire HAL, est destinée au dépôt et à la diffusion de documents scientifiques de niveau recherche, publiés ou non, émanant des établissements d'enseignement et de recherche français ou étrangers, des laboratoires publics ou privés. 


\title{
A TWO-DIMENSIONAL VERSION OF THE GODUNOV SCHEME FOR SCALAR BALANCE LAWS
}

\author{
LAURENT GOSSE*
}

\begin{abstract}
A Godunov scheme is derived for two-dimensional scalar conservation laws without or with source terms following ideas originally proposed by Boukadida and LeRoux [6] in the context of a staggered Lax-Friedrichs scheme. In both situations, the numerical fluxes are obtained at each interface of a uniform Cartesian computational grid just by means of the "external waves" involved in the entropy solution of the elementary $2 \mathrm{~d}$ Riemann problems; in particular, all the wave-interaction phenomena is overlooked. This restriction of the wave pattern suffices for deriving the exact numerical fluxes of the staggered LxF scheme, but it furnishes only an approximation for the Godunov scheme: we show that under convenient assumptions, these flux functions are smooth and the resulting discretization process is stable under nearly-optimal CFL restriction. A well-balanced extension is presented, relying on the Curl-free component of the Helmholtz decomposition of the source term. Several numerical tests against exact $2 \mathrm{D}$ solutions are performed for convex, non-convex and inhomogeneous equations and the time evolution of the $L^{1}$ truncation error is displayed.
\end{abstract}

Key words. Approximate 2D Riemann solver; External wave; Helmholtz decomposition and Curl-free field; Kružkov entropy solution; Well-Balanced 2D Godunov scheme.

AMS subject classifications. 65M06, 35L60.

1. Introduction. We are interested in deriving and proving rigorous estimates on a genuinely multi-dimensional Godunov scheme for a convex scalar balance law,

$$
\partial_{t} u+\partial_{x} f(u)+\partial_{y} g(u)=k(x, y) s(u), \quad x, y \in \mathbb{R}^{2}, \quad t>0,
$$

under the structural hypotheses (in particular, $s^{\prime}(u)$ has no definite sign):

$$
u(t=0, x, y)=u^{0}(x, y) \in L^{1} \cap L^{\infty} \cap B V\left(\mathbb{R}^{2}\right), \quad f^{\prime \prime}>0, \quad g^{\prime \prime}>0 .
$$

In spite of their simplicity, scalar models can be relevant in recent applications [12].

1.1. Overview of two-dimensional problems. Part of the success in the field of numerical approximation of one-dimensional (systems of) balance laws stems from the possibility of setting up a building block, the Riemann problem, which realizes the time-asymptotic behavior of the Cauchy problem. Thanks to strict hyperbolicity and entropy dissipation in shocks, the decay of Glimm's interaction potential implies that weak solutions evolve in time toward a non-interacting scattering state (e.g. in the sense of Liu-Yang [41]). However, in 2D, Riemann solutions don't systematically produce a non-interacting scattering state because corresponding wave patterns can still involve non-linear interactions: see for instance our Figures 4.7 and 4.8. The occurrence of such interactions complicate substantially the computation of these solutions $[40,55]$ which can hardly be set up as elementary building blocks in devising a $2 \mathrm{D}$ numerical scheme. Therefore, alternative derivations were proposed: see e.g. $[1,5,7,11,10,14,18,19,22$, $23,28,29,31,32,36,38,43,44,47,48,49,52]$.

The famous Kružkov uniqueness theorem ensures that there is a complete equivalence between the notion of entropy solutions and viscosity limits (see [16] for more details on this point) for multi-D scalar equations. This notwithstanding, the structure of the entropy solution of a scalar 2D Riemann problem displays an impressive complexity $[9,24,26,39,51,53,54]$ (see also $[15,50]$ for specific theoretical properties of multi-D scalar laws). However, within the framework of a 2D staggered Lax-Friedrichs scheme, Boukadida and LeRoux [6] observed that one doesn't need all the complexity of the wave pattern in order to derive the expression of the exact numerical fluxes. Indeed, since a staggered LxF scheme proceeds by taking locally space averages of the totality of

*IAC-CNR "MAURO PICONE" (SEZIONE DI ROMA), VIA DEI TAURINI 19, 00185 ROME (ITALY) L.GOSSE@BA.IAC.CNR.IT 
each Riemann fan, it suffices to know the so-called external waves (in the terminology of $[40,55])$, and forget about the nonlinear interactions occurring close to the origin: see Fig. 2.1 for an illustration. Advantages in such a construction were: stability under a CFL restriction, and genuine 2D numerical fluxes, meaning that the horizontal flux function perceives the vertical gradients too. This last property allows to accurately render delicate $2 \mathrm{D}$ phenomena which can be deleted by less sophisticate schemes which involve one-dimensional solvers only. However, such a staggered setup is not well-suited for computations in a bounded domain, thus it seems appealing to develop similar ideas in a more flexible Godunov framework. The main issue which must be circumvented in this program lies in the fact that Godunov fluxes are not taken outside the Riemann fan's interaction area, but along the axes passing through the origin: hence it is necessary to rely on an approximate Riemann solver, which should involve only computations of external waves.

Another motivation comes from the need to extend the methodology of well-balanced (WB) schemes [21] from the 1D scene toward the multi-dimensional one. On this side, the obstacle is that despite it is always possible to rewrite a source term $k(x) s(u)$ as a non-conservative product $s(u) \partial_{x} K(x)$, with $K$ defined up to a constant, this simple trick is ineffective in 2D. A way out can be found by noticing that the $1 \mathrm{D}$ case proceeds by applying the fundamental theorem of calculus, so it sounds reasonable to try to apply the same fundamental theorem, in its vectorial version though, in 2D: this is the Helmholtz decomposition of a smooth decaying vector field. This way, the source term showing up in (1.1) rewrites as $s(u)\left[\partial_{x} K_{x}+\partial_{y} K_{y}\right]$, with $\mathbf{K}:=\left(K_{x}, K_{y}\right)$ a gradient field which can be computed in a stable way numerically and then distributed inside both (horizontal or vertical) flux functions.

1.2. Organization of the paper. A basic object in the calculations presented hereafter is the two-dimensional version of the classical Lax-Friedrichs scheme published by Boukadida and LeRoux [6] (despite several inaccuracies in its presentation).

- In $\S 2$, a general construction of 2D Godunov numerical fluxes is presented, under the simplifying assumptions (2.5) and for the homogeneous case $k(x, y) \equiv 0$ first. In particular, all the derivations bypass the computation of nonlinear wave interactions occurring in the elementary Riemann problems. Only "external waves", that is to say, the one-dimensional Riemann waves appearing for $|x|,|y| \rightarrow \infty$ are used in the expression of our numerical fluxes (cf. (2.7) and $(2.8)$ ). These flux functions are shown to be smooth in $\S 2.2$. The subsection $\S 2.3$ presents the extension to the inhomogeneous case $k(x, y) \neq 0$ within the well-balanced framework, that is to say, by including the effects of the source term directly inside the expression of the numerical fluxes: see (2.16) and (2.17). These derivations make use of a Helmholtz decomposition of $(k, \mathbf{0})$ in order to distribute the source along each direction $O \vec{x}$ and $O \vec{y}$.

- In $\S 3$, some results about monotonicity properties of the resulting homogeneous 2D scheme are given under the hypotheses (2.5). By standard Crandall-Majda theory [13], this yields convergence toward the entropy solution as the grid parameters $\Delta x, \Delta y \rightarrow 0$ under a nearly optimal CFL restriction (2.1) for the time-step $\Delta t$. The more complex case involving wave interactions (3.3) is studied in $\S 3.3$ and the corresponding flux function is given in (3.6).

- In $\S 4$, various numerical results are provided, among which many involve an exact solution thanks to whom the actual $L^{1}$ errors can be measured, which allows to achieve accurate comparison with the more usual dimensional Strangsplitting scheme. Various simple 2D Riemann problems are simulated with both schemes and the time-evolution of resulting $L^{1}$ errors are displayed in $\S 4.1$. A "fingering test" taken from [25] is presented in $\S 4.2$. More involved 2D Riemann problems taken from both [53] and [28] are studied in $\S 4.3$; in particular, the exact solution given in [22] is used in order to scrutinize the 
onset of pointwise errors in both schemes, see Fig. 4.8. The $\S 4.4$ deals with (1.1) for which $f(u)=g(u)=\frac{u^{2}}{2}, k(x, y) \equiv 1$ and $s(u)=u$ : the WB scheme of $\S 2.3$ is presented and a test involving a rarefaction wave is investigated following the ideas of [3]. At last, $\S 4.5$ contains 3 non-convex benchmarks: the so-called "Guckenheimer structure" [5, 24, 54], the challenging "rotating KPP wave" $[22,31]$ and a Buckley-Leverett equation. Finally, some concluding remarks are given in $\S 5$.

2. Derivation of the two-dimensional numerical process. We start by defining a Cartesian uniform computational grid, determined by spatial parameters $\Delta x, \Delta y>$ 0 together with the time-step $\Delta t$ which is assumed to be small enough so as to satisfy the CFL condition:

$$
\max \left(\frac{\max \left|f^{\prime}\left(u^{0}\right)\right|}{\Delta x}, \frac{\max \left|g^{\prime}\left(u^{0}\right)\right|}{\Delta y}\right) \Delta t \leq 1,
$$

where the "max" is taken on the convex hull of all the values taken by the initial data $u^{0} \in L^{\infty}\left(\mathbb{R}^{2}\right)$. Elementary computational cells centered on $x_{i}=i \Delta x$ and $y_{j}=j \Delta y$, where the indexes $i, j \in \mathbb{Z}^{2}$, are denoted by $C_{i, j}$ : these are rectangles,

$$
C_{i, j}=\left(x_{i-\frac{1}{2}}, x_{i+\frac{1}{2}}\right) \times\left(y_{j-\frac{1}{2}}, y_{j+\frac{1}{2}}\right) .
$$

The numerical approximation $u^{\Delta t}$ is the piecewise constant function such that

$$
\forall i, j \in \mathbb{Z}^{2}, \quad u^{\Delta t}\left(t^{n}, x_{i}, y_{j}\right)=u_{i, j}^{n}
$$

on each elementary cell $C_{i, j}$ with $t^{n}=n \Delta t, n \in \mathbb{N}$.

2.1. A "nearly-exact" $2 \mathrm{D}$ Riemann solver in the convex case. Let us consider first the integral form of the homogeneous scalar conservation law (1.1): by integrating the equation on $\left(t^{n}, t^{n+1}\right) \times C_{i, j}$ one gets:

$$
\begin{aligned}
& \int_{C_{i, j}} u\left(t^{n+1}, x, y\right)-u\left(t^{n}, x, y\right) \cdot d x \cdot d y= \\
& \int_{t^{n}}^{t^{n+1}}\left(\int_{y_{j-\frac{1}{2}}}^{y_{j+\frac{1}{2}}}\left[f\left(u\left(\tau, x_{i-\frac{1}{2}}, y\right)\right)-f\left(u\left(\tau, x_{i+\frac{1}{2}}, y\right)\right)\right] d y\right. \\
&\left.\quad+\int_{x_{i-\frac{1}{2}}}^{x_{i+\frac{1}{2}}}\left[g\left(u\left(\tau, x, y_{j-\frac{1}{2}}\right)\right)-g\left(u\left(\tau, x, y_{j+\frac{1}{2}}\right)\right)\right] d x\right) d \tau .
\end{aligned}
$$

Hence, by denoting $u^{\Delta t}\left(0, x_{i}, y_{j}\right)=u_{i, j}^{0}=\int_{C_{i, j}} u^{0}(x, y) \frac{d x . d y}{\Delta x \Delta y} \simeq u^{0}\left(x_{i}, y_{j}\right)$ the value in the cell $C_{i, j}$, the time-marching process rewrite as a recursion formula involving numerical fluxes $F, G$ and emphasizing global mass conservation:

$$
u_{i, j}^{n+1}=u_{i, j}^{n}-\frac{\Delta t}{\Delta x}\left(F_{i+\frac{1}{2}, j}^{n}-F_{i-\frac{1}{2}, j}^{n}\right)-\frac{\Delta t}{\Delta y}\left(G_{i, j+\frac{1}{2}}^{n}-G_{i, j-\frac{1}{2}}^{n}\right) .
$$

Thanks to the piecewise-constant structure of $u^{\Delta t}\left(t^{n}, \cdot, \cdot\right)$, a convenient manner of computing the numerical fluxes along the interfaces of each cell proceeds by solving the Riemann problems occurring at each corner of the square (2.2). Accordingly, let $\omega$ be the self-similar entropy solution of these four-quadrant Riemann problems:

$$
\forall i, j \in \mathbb{Z}^{2}, \quad \omega_{i-\frac{1}{2}, j-\frac{1}{2}}^{n}\left(\frac{x-x_{i-\frac{1}{2}}}{\tau-t^{n}}, \frac{y-y_{j-\frac{1}{2}}}{\tau-t^{n}}\right), \quad \tau>0 .
$$


Indeed, assuming enough knowledge about $\omega$, it happens that:

$$
\begin{aligned}
F_{i-\frac{1}{2}, j}^{n}=\int_{0}^{\Delta t} & \left(\int_{y_{j-\frac{1}{2}}}^{y_{j}} f\left(\omega_{i-\frac{1}{2}, j-\frac{1}{2}}^{n}\left(0, \frac{y-y_{j-\frac{1}{2}}}{\tau}\right)\right) d y\right. \\
& +\int_{y_{j}}^{y_{j+\frac{1}{2}}} f\left(\omega_{i-\frac{1}{2}, j+\frac{1}{2}}^{n}\left(\left(0, \frac{y-y_{j+\frac{1}{2}}}{\tau}\right)\right) d y\right) \frac{d \tau}{\Delta t \Delta y},
\end{aligned}
$$

together with a similar expression for $G_{i, j-\frac{1}{2}}^{n}$. The basic observation which lies at the very heart of the Boukadida-LeRoux construction is that, within a staggered LaxFriedrichs scheme, only the restriction of $\omega$ on the boundary of staggered cells, the socalled external waves $[40,55]$, play a role in the computation of the exact numerical fluxes. Here, within a Godunov framework, only the restriction of all the Riemann solutions $\omega$ on the interfaces $x=x_{i \pm \frac{1}{2}}, y=y_{j \pm \frac{1}{2}}$ of the original cells $C_{i, j}$ must be taken into account. The original Boukadida-LeRoux idea stems on the fact that, in virtue
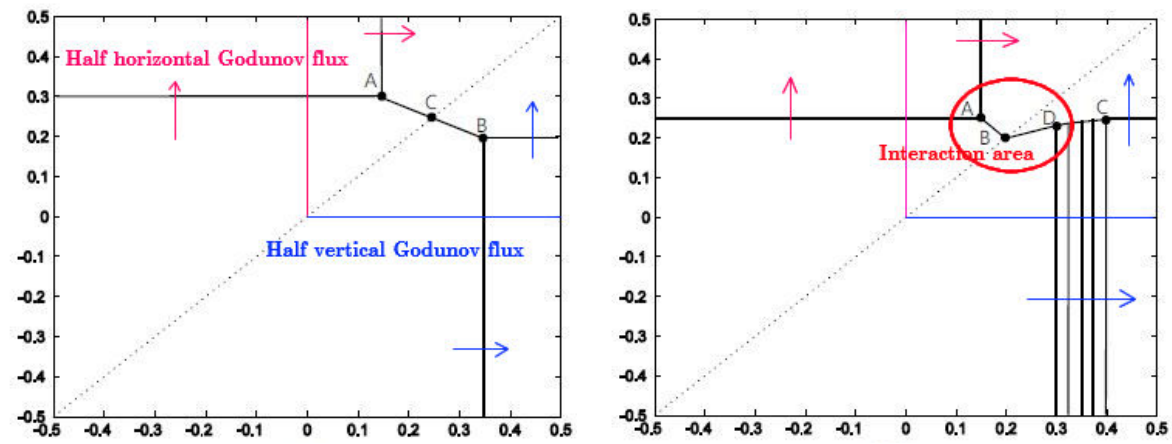

FIG. 2.1. Elementary 2D Riemann problems: "external waves" and interactions.

of the finite speed of propagation, restricting the CFL condition like (2.1) guarantees that the trace of the 2D Riemann solutions are given by transverse, or external, onedimensional Riemann problems (this fact is repeatedly used in $[24,51,54]$ ). Such an observation allows to considerably reduce the complexity in calculating numerical fluxes, because they essentially boil down to a time-average of a 1D Riemann fan. Now, the Godunov framework cannot allow for such a drastic simplification, but in many cases, the computation of integrals involved in e.g. (2.4) can still be achieved only by means of one-dimensional Riemann solutions, just containing the "external waves" and disregarding wave interactions. It is at this level that the convexity assumption on $f, g$ enters the picture: more precisely, it ensures that one-dimensional Riemann problems resulting from considering only either $\partial_{x} f(u)$ or $\partial_{y} g(u)$ in (1.1) contain just one wave which is an entropy shock or a rarefaction. To fix ideas, we hereafter assume

$$
f^{\prime}(0)=g^{\prime}(0)=0, \quad f^{\prime}, f^{\prime \prime}>0, g^{\prime}, g^{\prime \prime}>0 \text { in the domain of interest, }
$$

and proceed by distinguishing between 2 cases for $x$-fluxes $F_{i-\frac{1}{2}, j}^{n}$ :

- the "vertical" Riemann problem for the 1D scalar law:

$$
\partial_{t} v+\partial_{y} g(v)=0, \quad v\left(t=t^{n}, x, y\right)= \begin{cases}u_{i-1, j-1}^{n}, & y<y_{j-\frac{1}{2}} \\ u_{i-1, j}^{n}, & y>y_{j-\frac{1}{2}}\end{cases}
$$

yields an entropy shock (i.e. $u_{i-1, j-1}^{n}>u_{i-1, j}^{n}$ ) of Rankine-Hugoniot velocity,

$$
\sigma=\sigma_{i-1, j-\frac{1}{2}}^{n}=\frac{g\left(u_{i-1, j}^{n}\right)-g\left(u_{i-1, j-1}^{n}\right)}{u_{i-1, j}^{n}-u_{i-1, j-1}^{n}}>0 .
$$


By means of an elementary computation, the numerical flux (2.4) rewrites:

$$
\begin{aligned}
F_{i-\frac{1}{2}, j}^{n} & =\int_{0}^{\Delta t}\left(\int_{y_{j-\frac{1}{2}}}^{y_{j-\frac{1}{2}}+\tau \sigma} f\left(u_{i-1, j-1}^{n}\right) d y+\int_{y_{j-\frac{1}{2}}+\tau \sigma}^{y_{j+\frac{1}{2}}} f\left(u_{i-1, j}^{n}\right) d y\right) \frac{d \tau}{\Delta t \Delta y} \\
& =\int_{0}^{\Delta t}\left(\tau \sigma f\left(u_{i-1, j-1}^{n}\right)+(\Delta y-\tau \sigma) f\left(u_{i-1, j}^{n}\right)\right) \frac{d \tau}{\Delta t \Delta y} \\
& =\underbrace{f\left(u_{i-1, j}^{n}\right)}_{\text {normal (usual) component }}-\underbrace{\frac{\Delta t \sigma_{i-1, j-\frac{1}{2}}^{n}}{2 \Delta y}\left(f\left(u_{i-1, j}^{n}\right)-f\left(u_{i-1, j-1}^{n}\right)\right)}_{\text {tangential (anti-diffusive) component }}
\end{aligned}
$$

In most of upwind schemes, only the "normal component" is considered.

- the Riemann problem (2.6) is solved by means of a rarefaction wave:

$$
v(t, y)=\left\{\begin{array}{lr}
u_{i-1, j-1}^{n}, & \frac{y-y_{j-\frac{1}{2}}}{t-t^{n}}<g^{\prime}\left(u_{i-1, j-1}^{n}\right), \\
\left(g^{\prime}\right)^{-1}\left(\frac{y-y_{j-\frac{1}{2}}}{t-t^{n}}\right), & g^{\prime}\left(u_{i-1, j-1}^{n}\right) \leq \frac{y-y_{j-\frac{1}{2}}}{t-t^{n}} \leq g^{\prime}\left(u_{i-1, j}^{n}\right), \\
u_{i-1, j}^{n}, & \frac{y-y_{j-\frac{1}{2}}}{t-t^{n}}>g^{\prime}\left(u_{i-1, j}^{n}\right) .
\end{array}\right.
$$

The computation of the numerical flux (2.4) proceeds as follows:

$$
\begin{aligned}
F_{i-\frac{1}{2}, j}^{n}= & \int_{t^{n}}^{t^{n+1}}\left(\int_{y_{j-\frac{1}{2}}}^{y_{j-\frac{1}{2}}+g^{\prime}\left(u_{i-1, j-1}^{n}\right)\left(t-t^{n}\right)} f\left(u_{i-1, j-1}^{n}\right) d y\right. \\
& +\int_{y_{j-\frac{1}{2}}+t g^{\prime}\left(u_{i-1, j-1}^{n}\right)}^{y_{j+\frac{1}{2}}-t g^{\prime}\left(u_{i-1, j}^{n}\right)} f \circ\left(g^{\prime}\right)^{-1}\left(\frac{y-y_{j-\frac{1}{2}}}{t-t^{n}}\right) d y \\
& \left.+\int_{y_{j+\frac{1}{2}}-g^{\prime}\left(u_{i-1, j}^{n}\right)\left(t-t^{n}\right)}^{y_{j+\frac{1}{2}}} f\left(u_{i-1, j}^{n}\right) d y\right) \frac{d t}{\Delta t \Delta y}
\end{aligned}
$$

The middle term involves a change of variable,

$$
\int_{t^{n}}^{t^{n+1}} d t \int_{t g^{\prime}\left(u_{i-1, j-1}^{n}\right)}^{t g^{\prime}\left(u_{i-1, j}^{n}\right)} f \circ\left(g^{\prime}\right)^{-1}\left(\frac{y}{t-t^{n}}\right) d y=\int_{0}^{\Delta t} \tau d \tau \int_{u_{i-1, j-1}^{n}}^{u_{i-1, j}^{n}} f(v) g^{\prime \prime}(v) d v
$$

and this expression is handled by means of an integration by parts:

$$
=\frac{\Delta t^{2}}{2}\left(\left[f g^{\prime}\left(u_{i-1, j}^{n}\right)-f g^{\prime}\left(u_{i-1, j-1}^{n}\right)\right]-\int_{u_{i-1, j-1}^{n}}^{u_{i-1, j}^{n}} f^{\prime}(v) g^{\prime}(v) d v\right) .
$$

Summing the 3 integral terms, the numerical fluxes reduce to:

$$
F_{i-\frac{1}{2}, j}^{n}=\underbrace{f\left(u_{i-1, j}^{n}\right)}_{\text {normal (usual) component }}-\underbrace{\frac{\Delta t}{2 \Delta y} \int_{u_{i-1, j-1}^{n}}^{u_{i-1, j}^{n}} f^{\prime}(v) g^{\prime}(v) d v}_{\text {tangential (anti-diffusive) component }}
$$

All in all, the numerical flux in the $x$-direction reads:

$$
\begin{aligned}
& F_{i-\frac{1}{2}, j}^{n}=f\left(u_{i-1, j}^{n}\right)-\frac{\Delta t}{2 \Delta y}\left(\chi\left(u_{i-1, j-1}^{n}<u_{i-1, j}^{n}\right) \int_{u_{i-1, j-1}^{n}}^{u_{i-1, j}^{n}} f^{\prime}(v) g^{\prime}(v) d v+\right. \\
& \left.\chi\left(u_{i-1, j-1}^{n}>u_{i-1, j}^{n}\right) \frac{\left(f\left(u_{i-1, j}^{n}\right)-f\left(u_{i-1, j-1}^{n}\right)\right)\left(g\left(u_{i-1, j}^{n}\right)-g\left(u_{i-1, j-1}^{n}\right)\right)}{u_{i-1, j}^{n}-u_{i-1, j-1}^{n}}\right),
\end{aligned}
$$


together with the one in the $y$-direction (obtained through similar derivations),

$$
\begin{aligned}
& G_{i, j-\frac{1}{2}}^{n}=g\left(u_{i, j-1}^{n}\right)-\frac{\Delta t}{2 \Delta x}\left(\chi\left(u_{i-1, j-1}^{n}<u_{i, j-1}^{n}\right) \int_{u_{i-1, j-1}^{n}}^{u_{i, j-1}^{n}} f^{\prime}(v) g^{\prime}(v) d v+\right. \\
& \left.\chi\left(u_{i-1, j-1}^{n}>u_{i, j-1}^{n}\right) \frac{\left(f\left(u_{i, j-1}^{n}\right)-f\left(u_{i-1, j-1}^{n}\right)\right)\left(g\left(u_{i, j-1}^{n}\right)-g\left(u_{i-1, j-1}^{n}\right)\right)}{u_{i, j-1}^{n}-u_{i-1, j-1}^{n}}\right),
\end{aligned}
$$

where $\chi(A)$ stands for the characteristic function of the set $A$. One sees that, in the case under study, $F_{i-\frac{1}{2}, j}^{n}$ is indeed a function $\mathbb{R}^{2} \rightarrow \mathbb{R}$ which rewrites $F\left(u_{i-1, j-1}^{n}, u_{i-1, j}^{n}\right)$ too. The same statements obviously holds for $G_{i, j-\frac{1}{2}}^{n}=G\left(u_{i-1, j-1}^{n}, u_{i, j-1}^{n}\right)$.

REMARK 1. We stress that the aforementioned numerical process is not a version of the $N$-scheme [48] partly because all the integrals in time are exactly calculated; instead, one may recast it as a nonlinear version of both the CTU of Colella [11] and LeVeque's wave propagation algorithm [35], essentially derived in the context of advection equations. In this linear case where $f(u)=a u, g(u)=b u$, and $a, b>0$,

$$
\left.\begin{array}{l}
F_{i-\frac{1}{2}, j}^{n}=a\left(u_{i-1, j}^{n}\left(1-\frac{b \Delta t}{2 \Delta y}\right)+\frac{b \Delta t}{2 \Delta y} u_{i-1, j-1}^{n}\right) \\
G_{i, j-\frac{1}{2}}^{n}=b\left(u_{i, j-1}^{n}\left(1-\frac{a \Delta t}{2 \Delta x}\right)+\frac{a \Delta t}{2 \Delta x} u_{i-1, j-1}^{n}\right)
\end{array}\right\} .
$$

Its stencil is wider too, because it contains 4 points instead of 3 for the $N$-scheme. The (linear) numerical fluxes (2.9) already appeared in [35, 36] and §5.1 of [43]: high-order extensions are studied and Simpson's rule appears like being the most stable one.

2.2. Smoothness of the resulting numerical flux functions. As a first step toward a rigorous convergence proof of the scheme (2.3)-(2.7)-(2.8), we show that each numerical flux function $F, G$ is $C^{1}$ with respect to its 2 arguments. More precisely:

- let's consider the limit $u \rightarrow v, u<v$ (the "vertical rarefaction" in (2.6)),

$$
F(u, v)=f(v)-\frac{\Delta t}{2 \Delta y} \int_{u}^{v} f^{\prime}(\xi) g^{\prime}(\xi) d \xi \rightarrow f(v) .
$$

Considering the opposite direction, $u>v$ (the "vertical shock" in (2.6)),

$$
F(u, v)=f(v)-\frac{\Delta t}{2 \Delta y} \underbrace{\frac{f(v)-f(u)}{v-u}}_{\text {bounded }} \cdot \underbrace{\frac{g(v)-g(u)}{v-u}}_{\text {bounded }} \cdot(v-u) \rightarrow f(v) .
$$

Hence continuity holds on the diagonal $u=v$.

- the same procedure applied to the partial derivatives $\frac{\partial F}{\partial u / v}$ : for $u<v$,

$$
\nabla F(u, v)=\left(\frac{\Delta t}{2 \Delta y} f^{\prime}(u) g^{\prime}(u), \quad f^{\prime}(v)\left(1-\frac{\Delta t}{2 \Delta y} g^{\prime}(v)\right)\right)^{T},
$$

whereas, for $u>v$, a more intricate expression holds:

$$
\begin{aligned}
\nabla F(u, v)= & \left(\frac{\Delta t}{2 \Delta y} f^{\prime}(u) \frac{g(v)-g(u)}{v-u}-\frac{\Delta t}{2 \Delta y} \frac{f(v)-f(u)}{v-u}\left[\frac{g(v)-g(u)}{v-u}-g^{\prime}(u)\right],\right. \\
& \left.f^{\prime}(v)\left(1-\frac{\Delta t}{2 \Delta y} \frac{g(v)-g(u)}{v-u}\right)+\frac{\Delta t}{2 \Delta y} \frac{f(v)-f(u)}{v-u}\left[\frac{g(v)-g(u)}{v-u}-g^{\prime}(v)\right]\right)^{T}
\end{aligned}
$$

Consequently, $\nabla F(u, v)$ is continuous on the diagonal $u=v$, too. We have:

Lemma 2.1. Consider the scalar law (1.1) with convex fluxes $f, g$ which satisfy moreover (2.5), then the numerical fluxes $F, G$ defined by (2.7)-(2.8) are smooth and 
belong to $C^{1}\left(\mathbb{R}^{2} \rightarrow \mathbb{R}\right)$. Moreover, these numerical fluxes are consistent in the sense of Crandall-Majda with the continuous equation: $F(v, v)=f(v), G(v, v)=g(v)$.

For monotonicity properties, the following expression of $\nabla F$ with $v<u$ is useful:

$$
\begin{gathered}
\nabla F(u, v)=\left(\frac{\Delta t}{2 \Delta y}\{f^{\prime}(u) \frac{g(v)-g(u)}{v-u}+\frac{f(v)-f(u)}{v-u} \overbrace{\left[g^{\prime}(u)-\frac{g(v)-g(u)}{v-u}\right]}^{\geq 0 \text { for } v<u}\}^{T},\right. \\
\frac{f(v)-f(u)}{v-u}\left(1-\frac{\Delta t}{2 \Delta y} g^{\prime}(v)\right)+\underbrace{\left[f^{\prime}(v)-\frac{f(v)-f(u)}{v-u}\right]}_{\leq 0 \text { for } v<u}\left[1-\frac{\Delta t}{2 \Delta y} \frac{g(v)-g(u)}{v-u}\right])^{T}
\end{gathered}
$$

But one can write $\nabla F$ in a compact form too, i.e. like (2.10) plus corrective terms:

$$
\begin{aligned}
\nabla F(u, v)=\left(\frac{\Delta t}{2 \Delta y} f^{\prime}(u) g^{\prime}(u), \quad\right. & \left.f^{\prime}(v)\left(1-\frac{\Delta t}{2 \Delta y} g^{\prime}(v)\right)\right)^{T} \\
+\frac{\Delta t}{2 \Delta y} \chi(u>v) & \left(\left[f^{\prime}(u)-\frac{f(v)-f(u)}{v-u}\right]\left[\frac{g(v)-g(u)}{v-u}-g^{\prime}(u)\right],\right. \\
& {\left.\left[f^{\prime}(v)-\frac{f(v)-f(u)}{v-u}\right]\left[g^{\prime}(v)-\frac{g(v)-g(u)}{v-u}\right]\right)^{T}, }
\end{aligned}
$$

with similar formulas holding obviously for $\nabla G$.

2.3. Well-balanced extension to inhomogeneous equations. The assumptions (2.5) imply that (1.1) is in non-resonant regime and one can proceed by following standard considerations in 2D [21]. In the opposite (resonant) case, one can consult for instance $[37,27]$. One advantage of the preceding construction is that it doesn't ask for substantial modifications when source terms are added:

- Let's assume that we have at hand a smooth vector field $\mathbf{K}(x, y)$ satisfying:

$$
\forall x, y \in \mathbb{R}^{2}, \quad \nabla \cdot \mathbf{K}(x, y)=k(x, y), \quad \mathbf{K}=\left(K_{x}, K_{y}\right),
$$

with $\nabla \cdot$ standing for the divergence operator. Then (1.1) rewrites as follows:

$$
\partial_{t} u+\left[\partial_{x} f(u)-s(u) \partial_{x} K_{x}\right]+\left[\partial_{y} g(u)-s(u) \partial_{y} K_{y}\right]=0, \quad \partial_{t} \mathbf{K}=0 .
$$

The "steady vector" $\mathbf{K}$ can be discretized by means of a piecewise-constant approximation, denoted $\mathbf{K}^{\Delta}$, in a way similar as $u^{0}(x, y)$. The aforementioned equation, which appears to be a $2 \mathrm{D}$ version of the $2 \times 2$ system written in [27], becomes homogeneous, non-conservative in the sense of [33].

- To derive the conservative and irrotational field $\mathbf{K}$, we notice that there is no boundary condition imposed on (2.13), hence a solution exists: denote $\Delta$ the Laplace operator in $\mathbb{R}^{2}, \Delta^{-1}$ a right-inverse satisfying $\Delta \circ \Delta^{-1}=I d,[4,45]$

$$
\Delta \zeta=k, \quad \Rightarrow \quad \mathbf{K}=\nabla \zeta=\nabla\left(\Delta^{-1}[k]\right) .
$$

The solution $\nabla \zeta(x, y)$ just corresponds to the Curl-free component in the Helmholtz decomposition of $(k, \mathbf{0})$ since the null field is trivially solenoidal.

$$
\|\nabla \times \mathbf{K}\|=\left|\partial_{x} K_{y}-\partial_{y} K_{x}\right|=\left|\partial_{x y} \zeta-\partial_{y x} \zeta\right|=0 .
$$

As we are only interested in the jumps of $K_{x}^{\Delta}$ and $K_{y}^{\Delta}$ across the interfaces of the grid, we have a good candidate to mimic the classical one-dimensional wellbalanced approach [21]. Moreover, $\mathbf{K}$ is independent of the entropy solution $u(t, x, y)$ thus it bypasses the issue raised in [20] (see pp.146/147). 
- In order to practically compute reliable values of the conservative field $\mathbf{K}$ in a bounded computational domain, one must set up the Laplace equation of (2.14) with boundary conditions in such a manner that the corresponding finitedifferences matrix is invertible with a low condition number. Moreover, one needs to know both $K_{x}^{\Delta}(\cdot, y)$ in all the central nodes $x_{i}$ 's, and $K_{y}^{\Delta}(x, \cdot)$ in all the $y_{j}$ 's of the computational domain. One way to achieve these goals is to impose Dirichlet boundary conditions on $\zeta$ and to solve for $k\left(x_{i-\frac{1}{2}}, y_{j-\frac{1}{2}}\right)$, that is, at the vertices of the computational cells. This way, the components $K_{x}^{\Delta}, K_{y}^{\Delta}$ which are divided differences are known at the right locations, i.e. at the edges:

$$
K_{x}^{\Delta}\left(x_{i}, y_{j-\frac{1}{2}}\right)=\frac{\zeta\left(x_{i+\frac{1}{2}}, y_{j-\frac{1}{2}}\right)-\zeta\left(x_{i-\frac{1}{2}}, y_{j-\frac{1}{2}}\right)}{\Delta x},
$$

and similarly,

$$
K_{y}^{\Delta}\left(x_{i-\frac{1}{2}}, y_{j}\right)=\frac{\zeta\left(x_{i-\frac{1}{2}}, y_{j+\frac{1}{2}}\right)-\zeta\left(x_{i-\frac{1}{2}}, y_{j-\frac{1}{2}}\right)}{\Delta y} .
$$

The WB version of the Godunov scheme (2.3) under the assumptions (2.5) reads:

$$
u_{i, j}^{n+1}=u_{i, j}^{n}-\frac{\Delta t}{\Delta x}\left(F_{i+\frac{1}{2}, j}^{n}-\tilde{F}_{i-\frac{1}{2}, j}^{n}\right)-\frac{\Delta t}{\Delta y}\left(G_{i, j+\frac{1}{2}}^{n}-\tilde{G}_{i, j-\frac{1}{2}}^{n}\right) .
$$

Formal consistency with the original equation (1.1) is ensured as soon as there holds:

$$
\frac{1}{\Delta x}\left(\tilde{F}_{i-\frac{1}{2}, j}^{n}-F_{i-\frac{1}{2}, j}^{n}\right)+\frac{1}{\Delta y}\left(\tilde{G}_{i-\frac{1}{2}, j}^{n}-G_{i-\frac{1}{2}, j}^{n}\right)=k\left(x_{i}, y_{j}\right) s(u)+R_{i, j}^{n},
$$

with the remainder $\left|R_{i, j}^{n}\right| \rightarrow 0$ when $\Delta x, \Delta y \rightarrow 0$. In conjunction with the approximation of 2 D Riemann problems with "external waves" only, there exist at least 2 ways (of increasing difficulty) to compute the modified WB fluxes:

1. (1D treatment of source term), from (2.7), one derives

$$
\left\{\begin{array}{l}
\tilde{F}_{i-\frac{1}{2}, j}^{n}:=f\left(\tilde{u}_{x, i-1, j}^{n}\right)-\frac{\Delta t}{2 \Delta y}(\ldots \text { tangential terms ...), } \\
\tilde{G}_{i, j-\frac{1}{2}}^{n}:=g\left(\tilde{u}_{y, i, j-1}^{n}\right)-\frac{\Delta t}{2 \Delta x}(\ldots \text { tangential terms ...), }
\end{array}\right.
$$

where the "interface values" are defined according to,

$$
\left\{\begin{array}{l}
\tilde{u}_{x, i-1, j}^{n}=\phi^{-1}\left(\phi\left(u_{i-1, j}^{n}\right)-\left[K_{x}\left(x_{i}, y_{j-\frac{1}{2}}\right)-K_{x}\left(x_{i-1}, y_{j-\frac{1}{2}}\right)\right]\right), \\
\phi^{\prime}(u):=\frac{f^{\prime}(u)}{s(u)}
\end{array}\right.
$$

and, similarly,

$$
\left\{\begin{array}{l}
\tilde{u}_{y, i, j-1}^{n}=\psi^{-1}\left(\psi\left(u_{i, j-1}^{n}\right)-\left[K_{y}\left(x_{i-\frac{1}{2}}, y_{j}\right)-K_{y}\left(x_{i-\frac{1}{2}}, y_{j-1}\right)\right]\right), \\
\psi^{\prime}(u):=\frac{g^{\prime}(u)}{s(u)} .
\end{array}\right.
$$

For such a choice (only the "usual terms" are modified), formal consistency can be established by means of one-dimensional considerations because, if $v$ stands for the integral curve of $\partial_{x} f(v)=\partial_{x} K_{x}\left(x, y_{j-\frac{1}{2}}\right) s(v)$, then,

$$
f\left(\tilde{u}_{x, i-1, j}^{n}\right)=f\left(u_{i-1, j}^{n}\right)+\int_{x_{i-1}}^{x_{i}} s(v) \partial_{x} K_{x}\left(\xi, y_{j-\frac{1}{2}}\right) d \xi
$$

and a similar jump relation holds for $g\left(\tilde{u}_{y, i, j-1}^{n}\right)$. By construction, it holds that $\partial_{x} K_{x}+\partial_{y} K_{y}=\Delta \zeta=k$, hence one recovers the correct coefficient on $s$. 
2. (full 2D treatment of source term), Based on the smooth functions $F, G$, one defines modified fluxes in which jump relations are imposed in both directions

$$
\left\{\begin{array}{l}
\tilde{F}_{i-\frac{1}{2}, j}^{n}:=F\left(\tilde{u}_{y, i-1, j-1}^{n}, \tilde{u}_{x, i-1, j}^{n}\right) \\
\tilde{G}_{i, j-\frac{1}{2}}^{n}:=G\left(\tilde{u}_{x, i-1, j-1}^{n}, \tilde{u}_{y, i, j-1}^{n}\right)
\end{array}\right.
$$

where, according to the same functions $\phi^{\prime}(u):=\frac{f^{\prime}(u)}{s(u)}, \psi^{\prime}(u):=\frac{g^{\prime}(u)}{s(u)}$,

$$
\left\{\begin{array}{l}
\tilde{u}_{y, i-1, j-1}^{n}=\psi^{-1}\left(\psi\left(u_{i-1, j-1}^{n}\right)-\left[K_{y}\left(x_{i-\frac{1}{2}}, y_{j}\right)-K_{y}\left(x_{i-\frac{1}{2}}, y_{j-1}\right)\right]\right), \\
\tilde{u}_{x, i-1, j-1}^{n}=\phi^{-1}\left(\phi\left(u_{i-1, j-1}^{n}\right)-\left[K_{x}\left(x_{i}, y_{j-\frac{1}{2}}\right)-K_{x}\left(x_{i-1}, y_{j-\frac{1}{2}}\right)\right]\right) .
\end{array}\right.
$$

Both these "tangential interface values" were missing in the former simplified derivation. With these more involved fluxes, formal consistency of modified WB scheme (2.15) is far less clear. However, the corresponding numerical results are more accurate: see $\S 4.4$. A meaningful question is whether or not the gain in terms of accuracy seen in 1D [3,21] still holds in 2D (see also [2]).

\section{Monotonicity, BV bounds and strong compactness results.}

3.1. Monotonicity under the restriction (2.5). In the homogeneous case (no source terms), one has the following important property:

Lemma 3.1. Assume that (2.5) and one of the CFL conditions holds true,

- (2.1) in the case only rarefaction waves occur in the "tangential terms",

- in the general case,

$$
\left(\frac{\max \left|f^{\prime}\left(u^{0}\right)\right|}{\Delta x}+\frac{\max \left|g^{\prime}\left(u^{0}\right)\right|}{\Delta y}\right) \Delta t \leq 1,
$$

then the 2D scheme (2.3)-(2.7)-(2.8) is monotone in the sense of Crandall-Majda. In particular, it satisfies the maximum principle and both its $L^{1}\left(\mathbb{R}^{2}\right)$ norm and total variation decay when $n \in \mathbb{N}$ grows:

$$
T V\left(u^{\Delta t}\left(t^{n}, \cdot, \cdot\right)\right)=\sum_{i, j \in \mathbb{Z}^{2}} \Delta y\left|u_{i, j}^{n}-u_{i-1, j}^{n}\right|+\Delta x\left|u_{i, j}^{n}-u_{i, j-1}^{n}\right| \leq T V\left(u^{0}\right) .
$$

Proof. The 2D Godunov scheme reads for all $i, j, n \in \mathbb{Z} \times \mathbb{Z} \times \mathbb{N}$ :

$$
\begin{aligned}
u_{i, j}^{n+1}=u_{i, j}^{n}-\Delta t & {\left[\left(\frac{F\left(u_{i, j-1}^{n}, u_{i, j}^{n}\right)}{\Delta x}+\frac{G\left(u_{i-1, j}^{n}, u_{i, j}^{n}\right)}{\Delta y}\right)\right.} \\
& \left.-\left(\frac{F\left(u_{i-1, j-1}^{n}, u_{i-1, j}^{n}\right)}{\Delta x}+\frac{G\left(u_{i-1, j-1}^{n}, u_{i, j-1}^{n}\right)}{\Delta y}\right)\right] .
\end{aligned}
$$

One thus defines the following smooth function $\mathcal{G}: \mathbb{R}^{4} \rightarrow \mathbb{R}$, such that:

$$
\begin{aligned}
u_{i, j}^{n+1}= & \mathcal{G}\left(u_{i-1, j-1}^{n}, u_{i-1, j}^{n}, u_{i, j-1}^{n}, u_{i, j}^{n}\right) \\
= & u_{i, j}^{n}-\Delta t\left[\mathcal{F}\left(u_{i, j-1}^{n}, u_{i, j}^{n}, u_{i-1, j}^{n}, u_{i, j}^{n}\right)\right. \\
& \left.\quad-\mathcal{F}\left(u_{i-1, j-1}^{n}, u_{i-1, j}^{n}, u_{i-1, j-1}^{n}, u_{i, j-1}^{n}\right)\right] .
\end{aligned}
$$

The monotonicity in the sense of Crandall-Majda [13] means that $\mathcal{G}$ is a non-decreasing function of all its arguments. We can proceed by examining all its partial derivatives, for both the cases where a rarefaction or a shock appears somewhere inside the numerical fluxes. The idea is to alternate between both the expressions (2.12) and (2.11) in order to let positive quantities appear where they are needed. 
- let's look first at the dependence in $u_{i, j}^{n}$ in (3.2), we aim at showing:

$$
\frac{\partial \mathcal{G}}{\partial u_{i, j}^{n}}=1-\left.\Delta t\left(\frac{1}{\Delta x} \frac{\partial F}{\partial v}+\frac{1}{\Delta y} \frac{\partial G}{\partial v}\right)\right|_{v=u_{i, j}^{n}} \geq 0 .
$$

Suppose first that both $\frac{\partial F}{\partial v}$ and $\frac{\partial G}{\partial v}$ involve a rarefaction wave inside the "tangential terms": under the less demanding CFL restriction (2.1), one gets by means of (2.10) that,

$1-\frac{\Delta t}{\Delta x} f^{\prime}\left(u_{i, j}^{n}\right)-\frac{\Delta t}{\Delta y} g^{\prime}\left(u_{i, j}^{n}\right)+\frac{\Delta t^{2}}{\Delta x \Delta y} f^{\prime} g^{\prime}\left(u_{i, j}^{n}\right)=\left(1-\frac{\Delta t}{\Delta x} f^{\prime}\left(u_{i, j}^{n}\right)\right)\left(1-\frac{\Delta t}{\Delta y} g^{\prime}\left(u_{i, j}^{n}\right)\right) \geq 0$.

Oppositely, if $\frac{\partial F}{\partial v}$ involves an entropy shock inside its "tangential terms", it is necessary that $u_{i, j}^{n}<u_{i, j-1}^{n}$, and by convexity of $f$, one gets:

$$
f^{\prime}\left(u_{i, j}^{n}\right) \leq \frac{f\left(u_{i, j}^{n}\right)-f\left(u_{i, j-1}^{n}\right)}{u_{i, j}^{n}-u_{i, j-1}^{n}} \leq f^{\prime}\left(u_{i, j-1}^{n}\right) .
$$

Hence we take advantage of (2.11) to obtain, with the stringent CFL (3.1):

$$
\begin{gathered}
1-\frac{\Delta t}{\Delta x} \frac{f\left(u_{i, j}^{n}\right)-f\left(u_{i, j-1}^{n}\right)}{u_{i, j}^{n}-u_{i, j}^{n}-1}\left(1-\frac{\Delta t}{2 \Delta y} g^{\prime}\left(u_{i, j}^{n}\right)\right)-\frac{\Delta t}{\Delta y} g^{\prime}\left(u_{i, j}^{n}\right)\left(1-\frac{\Delta t}{2 \Delta x} f^{\prime}\left(u_{i, j}^{n}\right)\right) \\
+\frac{\Delta t}{\Delta x}\left(\frac{f\left(u_{i, j}^{n}\right)-f\left(u_{i, j-1}^{n}\right)}{u_{i, j}^{n}-u_{i, j}^{n}-1}-f^{\prime}\left(u_{i, j}^{n}\right)\right)\left(1-\frac{\Delta t}{2 \Delta y} \frac{g\left(u_{i, j}^{n}\right)-g\left(u_{i, j-1}^{n}\right)}{u_{i, j}^{n}-u_{i, j-1}^{n}}\right) \\
\geq 1-\Delta t\left(\frac{1}{\Delta x} \frac{f\left(u_{i, j}^{n}\right)-f\left(u_{i, j-1}^{n}\right)}{u_{i, j}^{n}-u_{i, j-1}^{n}}+\frac{1}{\Delta y} g^{\prime}\left(u_{i, j}^{n}\right)\right) \geq 0 .
\end{gathered}
$$

The case where $\frac{\partial G}{\partial v}$ too contains a shock is similar, thus $\frac{\partial \mathcal{G}}{\partial u_{i, j}^{n}} \geq 0$.

- from (3.2), the dependence on $u_{i-1, j-1}^{n}$ is still quite easy:

$$
\frac{\partial \mathcal{G}}{\partial u_{i-1, j-1}^{n}}=\left.\Delta t\left(\frac{1}{\Delta x} \frac{\partial F}{\partial u}+\frac{1}{\Delta y} \frac{\partial G}{\partial u}\right)\right|_{u=u_{i-1, j-1}^{n}}
$$

The case of rarefaction waves inside both the "tangential terms" is obvious. Then, we focus on $\frac{\partial F}{\partial u}$ and suppose there is a shock in those terms: again, the expression (2.11) leads to:

$$
\begin{aligned}
0 \leq & f^{\prime}\left(u_{i-1, j-1}^{n}\right) \frac{g\left(u_{i-1, j}^{n}\right)-g\left(u_{i-1, j-1}^{n}\right)}{u_{i-1, j}^{n}-u_{i-1, j-1}^{n}} \\
& +\frac{f\left(u_{i-1, j}^{n}\right)-f\left(u_{i-1, j-1}^{n}\right)}{u_{i-1, j}^{n}-u_{i-1, j-1}^{n}} \underbrace{\left[g^{\prime}\left(u_{i-1, j-1}^{n}\right)-\frac{g\left(u_{i-1, j}^{n}\right)-g\left(u_{i-1, j-1}^{n}\right)}{\left.u_{i-1, j}^{n}-u_{i-1, j-1}^{n}\right]}\right.}_{\geq 0 \text { for } u_{i-1, j}^{n}<u_{i-1, j-1}^{n}} .
\end{aligned}
$$

Since all the terms are endowed with the right sign, the situation will be identical for $\frac{\partial G}{\partial u}$ thus we are done with showing that $\frac{\partial \mathcal{G}}{\partial u_{i-1, j-1}^{n}} \geq 0$.

- in order to handle the next partial derivative,

$$
\frac{\partial \mathcal{G}}{\partial u_{i, j-1}^{n}}=-\Delta t\left(\left.\frac{1}{\Delta x} \frac{\partial F}{\partial u}\right|_{u=u_{i, j-1}^{n}}-\left.\frac{1}{\Delta y} \frac{\partial G}{\partial v}\right|_{v=u_{i, j-1}^{n}}\right),
$$

it is now necessary to use (2.12), that is to say, the expression of $\nabla F$ corresponding to a "tangential rarefaction wave" plus some corrective terms. Indeed, for $\frac{\partial F}{\partial u}$, the convexity of $f$ ensures that either this correction term vanishes (if $u_{i, j-1}^{n} \leq u_{i, j}^{n}$ ), either it is negative (if $u_{i, j-1}^{n}>u_{i, j}^{n}$ ). In exactly the same manner, the corrective term for $\frac{\partial G}{\partial v}$ vanish or is positive. Hence the corrective terms 
appearing in $\frac{\partial \mathcal{G}}{\partial u_{i, j-1}^{n}}$ if a "tangential shock" occurs in either $F$ or $G$ have all the good sign, i.e. they are nonnegative. Now, it is easy to see that the terms corresponding to (2.10) balance each other:

$$
\begin{array}{r}
\frac{1}{\Delta t} \frac{\partial \mathcal{G}}{\partial u_{i, j-1}^{n}}=-f^{\prime}\left(u_{i, j-1}^{n}\right)\left(\frac{1}{\Delta x} \frac{\Delta t}{2 \Delta y} g^{\prime}\left(u_{i, j-1}^{n}\right)-\frac{1}{\Delta y}\left[1-\frac{\Delta t}{2 \Delta x} g^{\prime}\left(u_{i, j-1}^{n}\right)\right]\right) \\
=\frac{f^{\prime}\left(u_{i, j-1}^{n}\right)}{\Delta y}\left(1-\frac{\Delta t}{\Delta x} g^{\prime}\left(u_{i, j-1}^{n}\right)\right) \geq 0 .
\end{array}
$$

- at last, the last partial derivative reads:

$$
\frac{\partial \mathcal{G}}{\partial u_{i-1, j}^{n}}=-\Delta t\left(\left.\frac{1}{\Delta y} \frac{\partial G}{\partial u}\right|_{u=u_{i-1, j}^{n}}-\left.\frac{1}{\Delta x} \frac{\partial F}{\partial v}\right|_{v=u_{i-1, j}^{n}}\right) .
$$

Positivity is shown proceeding exactly as before, so computations are skipped.

0

Numerical results in $\S 4$, see for instance the "fingering benchmark" displayed on Fig. 4.4 and the Yoon-Hwang 4-shocks test on Fig. 4.4, both realized with a Courant number of 0.95 strongly suggest that monotonicity holds in the presence of shocks even under the weak CFL restriction (2.1) if (2.5) is satisfied.

3.2. Monotonicity, entropy consistency and convergence. We are now in position to invoke a classical convergence criterion (see [13], Theorem 1):

THEOREM 1. The sequence of numerical approximations $u^{\Delta t}$ generated by the scheme (2.3)-(2.7)-(2.8) converges strongly in $L_{\text {loc }}^{1}\left(\mathbb{R}_{*}^{+} \times \mathbb{R}^{2}\right)$, as $\Delta t, \Delta x, \Delta y \rightarrow 0$ under one of the CFL conditions shown in Lemma 3.1, toward the unique entropy solution of (1.1)-(1.2) with fluxes satisfying (2.5).

Proof. Requirements of Crandall-Majda's result are a monotone scheme in conservation form, with smooth and consistent numerical fluxes. Based on the expression (2.3), the Lemmas 2.1 and 3.1, the only condition still to be checked is the sufficiency of the CFL restrictions given at $t=0$ : this is indeed a consequence of the maximum principle in $L^{\infty}$ and the smoothness of the exact (and convex) fluxes $f, g$.

3.3. Nonlinear wave interactions for the general case. For smooth solutions, it is well-known that truncation errors in linear one-dimensional problems are of the order of $\Delta x(1-\nu) t$ when $\nu$ stands for the Courant number (see $\S 2$ in [3]). Hence, it can be appealing, in terms of numerical accuracy, to study $2 \mathrm{D}$ numerical fluxes with more compressive cases for which the assumptions (2.5) are relaxed. Here we shall consider a slight extension, namely (1.1)-(1.2) where $g^{\prime}$ has no definite sign:

$$
f^{\prime}(0)=g^{\prime}(0)=0, \quad f^{\prime}, f^{\prime \prime}>0, g^{\prime \prime}>0 \text { in the domain of interest. }
$$

The only way to allow the Courant number to go beyond $\frac{1}{2}$ is to stipulate how the tangential part of $F_{i-\frac{1}{2}, j}^{n}$ is modified inside the computational cells for which strong compression occurs, that is to say, data enters from both the bottom and the top. As we still assume that $f^{\prime}>0$ has a definite sign, only the normal component of $G_{i, j-\frac{1}{2}}^{n}$ should be modified in order to cope with the local upwinding process (because no strong compression can occur in the horizontal direction). For easiness in writing, we show how to compute $F_{i+\frac{1}{2}, j}^{n}$ and define the "vertical shock" velocities for (2.6):

$$
\sigma_{ \pm}=\frac{g\left(u_{i, j \pm 1}^{n}-g\left(u_{i, j}^{n}\right)\right.}{u_{i, j \pm 1}^{n}-u_{i, j}^{n}}, \quad \mp \sigma_{ \pm}>0 .
$$

When the Courant number is smaller than $\frac{1}{2}$, these two vertical shocks cannot interact before $t^{n+1}$, but such a restriction amplifies numerical dissipation. The advantage is 
that $x$-fluxes still have a simple expression involving elementary integrals in time:

$F_{i+\frac{1}{2}, j}^{n}=\int_{0}^{\Delta t}\left(\int_{y_{j-\frac{1}{2}}}^{y_{j-\frac{1}{2}+\tau \sigma_{-}}} f\left(u_{i, j-1}^{n}\right) d y+\int_{y_{j-\frac{1}{2}+\tau \sigma_{-}}}^{y_{j+\frac{1}{2}}+\tau \sigma_{+}} f\left(u_{i, j}^{n}\right) d y+\int_{y_{j+\frac{1}{2}+\tau \sigma_{+}}}^{y_{j+\frac{1}{2}}} f\left(u_{i, j+1}^{n}\right) d y\right) \frac{d \tau}{\Delta t \Delta y}$.

However, for a Courant number greater than $\frac{1}{2}$, there always exists an interaction time $\tau^{*}<\Delta t$ at which incident shocks meet and merge (here the convexity of $g$ plays a role). We use some cell-dependent notation:

$$
\tau^{*}=\frac{\Delta y}{\sigma_{-}-\sigma_{+}}, \quad \tilde{\sigma}=\frac{g\left(u_{i, j+1}^{n}\right)-g\left(u_{i, j-1}^{n}\right)}{u_{i, j+1}^{n}-u_{i, j-1}^{n}} .
$$

Clearly, $\tilde{\sigma}$ is the velocity of the entropy shock emerging from the interaction in:

$$
y^{*}=y_{j+\frac{1}{2}}+\tau^{*} \sigma_{+}=y_{j-\frac{1}{2}}+\tau^{*} \sigma_{-} \text {at time } \tau^{*} .
$$

The former expression for $F_{i+\frac{1}{2}, j}^{n}$ still holds but the bounds of the time-integral become 0 and $\tau^{*}<\Delta t$, thus we don't rewrite it. New terms, corresponding to $\tau>\tau^{*}$ read:

$$
\int_{0}^{\Delta t-\tau^{*}}\left(\int_{y_{j-\frac{1}{2}}}^{y^{*}+\tau \tilde{\sigma}} f\left(u_{i, j}^{n}\right) d y+\int_{y^{*}+\tau \tilde{\sigma}}^{y_{j+\frac{1}{2}}} f\left(u_{i, j+1}^{n}\right) d y\right) \frac{d \tau}{\Delta t \Delta y}
$$

The "usual" normal component of the $x$-fluxes completely disappear from this expression, showing that our 2D approach strongly differ from more conventional discretizations especially for benchmarks involving delicate multi-dimensional wave interactions. The detailed computation of the integral terms is not reproduced here, but it is rather straightforward. Finally, the expression of the 2D $x$-fluxes handling vertical shock interactions is as follows:

$$
\begin{aligned}
F_{i+\frac{1}{2}, j}^{n}=\frac{1}{2 \Delta t}\left\{\tau^{*} f\left(u_{i, j}^{n}\right)\right. & +\frac{f\left(u_{i, j-1}^{n}\right)}{\Delta y}\left[\sigma_{-} \tau^{*}\left(2 \Delta t-\tau^{*}\right)+\tilde{\sigma}\left(\Delta t-\tau^{*}\right)^{2}\right] \\
& \left.-\frac{f\left(u_{i, j+1}^{n}\right)}{\Delta y}\left[\sigma_{+} \tau^{*}\left(2 \Delta t-\tau^{*}\right)+\tilde{\sigma}\left(\Delta t-\tau^{*}\right)^{2}\right]\right\}
\end{aligned}
$$

with all the necessary parameters given by (3.4) and (3.5). These seemingly intricate numerical fluxes were tested in several 2D benchmarks with strictly convex fluxes $f, g$ : see Figs. 4.4 and 4.7. One may be tempted to use them on non-convex problems, like the ones in Buckley-Leverett equations, but they can produce entropy-violating solutions for a CFL number greater than $\frac{1}{2}$ : see e.g. the bottom of Fig. 4.13.

4. Numerical results. In all the forthcoming benchmarks, the numerical scheme (2.3)-(2.7)-(2.8) is compared to a second-order dimensional Strang-splitting (as advocated in [13]) because the classical first-order 5-point upwind scheme is much too crude and its discrepancies with respect to the present 2D discretization are too important.

4.1. Diagonal benchmarks for homogeneous equations. For all "diagonal" benchmarks, both the exact flux functions are equal: $f(u)=g(u)=\frac{u^{2}}{2}$, which allows to derive exact solutions and scrutinize accurately the time-evolution of truncation errors. The first (and rather elementary) test is displayed on Fig. 4.1: it consists in choosing initial data which lead to an entropy solution taking the form of a diagonal rarefaction wave. More precisely, one picks up $u^{0}(x, y)=\chi(x+0.8>0) \chi(y+0.8>0)$ on the square $x, y \in(-1,1)$. Both schemes are run until $t=1.5$ with a Courant number of 0.95. As time grows, a slightly stronger $L^{1}$ error develops in the Strang-split scheme with respect to our 2D Godunov scheme (the exact solution is given in $\S 3.1$ of [5]): see the graphic on the right of Fig. 4.1. Let us explain the reason for this discrepancy: 

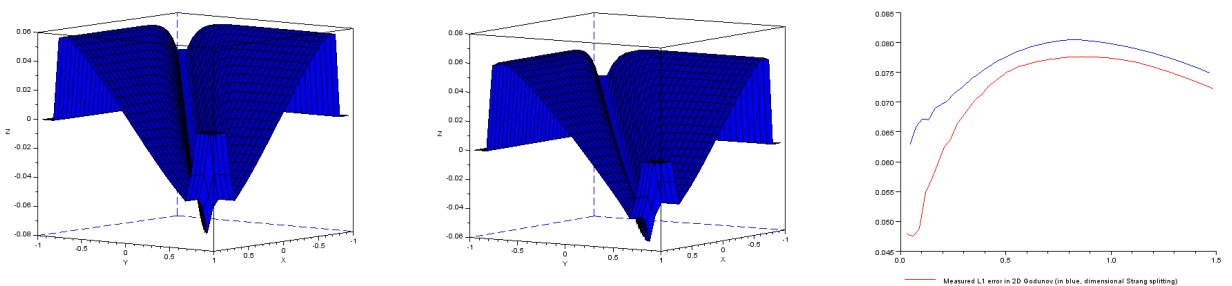

FIG. 4.1. Abolute error with exact rarefaction (2D Godunov, Strang-split, in time; left to right).

by inspecting the graphic middle of Fig. 4.1, one sees that the pointwise error of the dimensional Strang-split scheme is not symmetric with respect to the diagonal $x=y$. In sharp contrast, the pointwise error generated by the 2D Godunov scheme is perfectly symmetric (graphic on the left). Such a drawback is inherent to any splitting method. The opposite test-case is the one of the entropy shock emerging from initial data of the
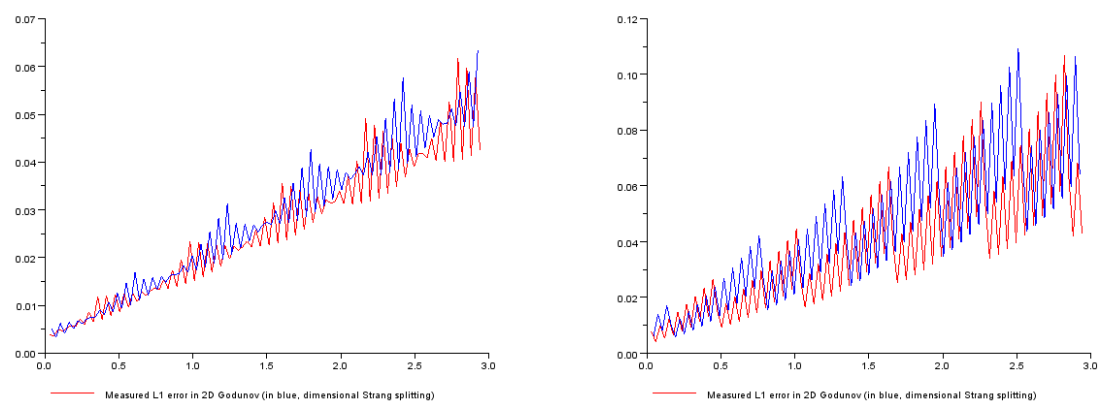

FIG. 4.2. Time evolutions of $L^{1}$-error for 2D-Godunov (red) and Strang-split (blue).

type $u^{0}(x, y)=\chi\left(x+x_{0}<0\right) \chi\left(y+y_{0}<0\right)$ : the graphics in Fig. 4.2 display the timeevolution of the $L^{1}$ error (with respect to the exact solution) of our 2D Godunov scheme compared to the one of the Strang dimensional splitting. Left/right graphics correspond to $x_{0}=y_{0}=0.8$ and 0.85 , respectively. There are $2^{6}$ points in each $x, y$-direction, which gives $\Delta x=\Delta y=2^{-5}$ for a CFL number of 0.95 .
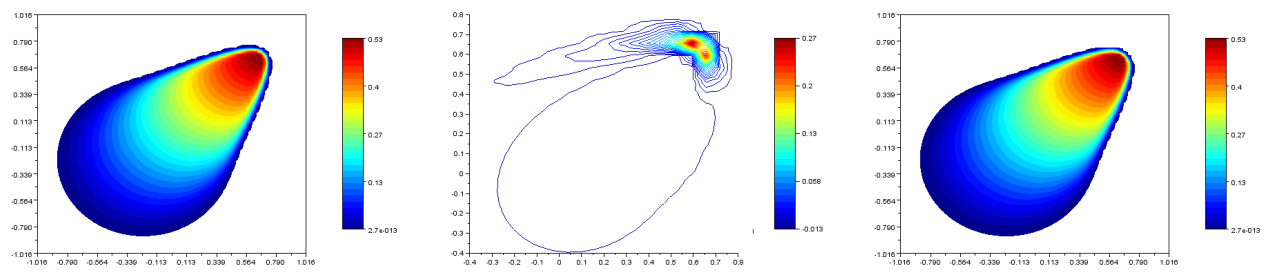

FIG. 4.3. Gaussian initial data: 2D Godunov (left), difference (middle), Strang-split (right).

4.2. Two cases involving circular initial data. For the next two test-cases, no exact solution seems to be available, but a good empirical knowledge of the entropy solution exists. The first case consists in considering simply $f(u)=g(u)=\frac{u^{2}}{2}$ with the Gaussian initial data $u^{0}(x, y)=\exp \left(-10\left((x+0.25)^{2}+(y+0.25)^{2}\right)\right), x, y \in(-1,1)$. Both numerical schemes are iterated until $t=1.3$ with the same computational grid $\Delta x=\Delta y=2^{-5}$ for a CFL number of 0.95. The graphic in the middle of Fig. 4.3 shows that differences between these approaches appear mainly in the vicinity of the 
spike: in particular, the approximation delivered by the 2D Godunov scheme is "more symmetric" with respect to the diagonal $x=y$. This second test-case is taken from the
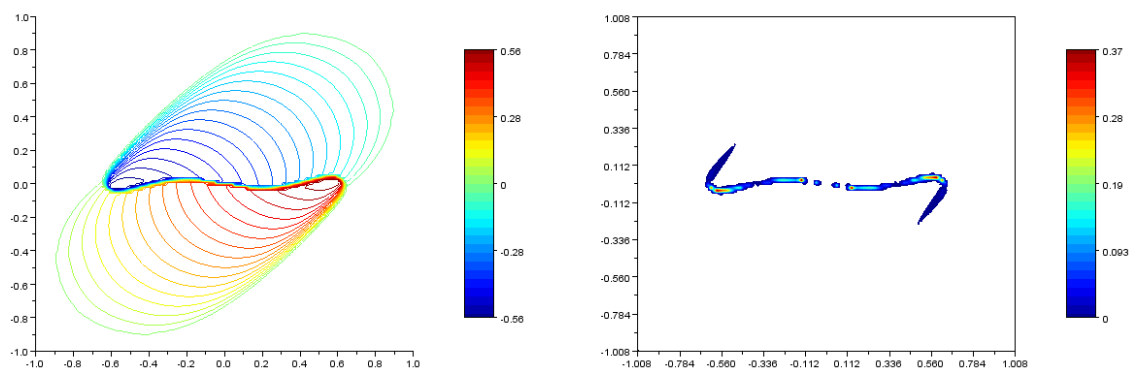

FIG. 4.4. Fingering test (CFL=0.95): 2D Godunov (left), difference with Strang-split (right).

book [25], pages 109/110. It involves slightly different flux functions,

$$
f(u)=\frac{u^{2}}{2}, \quad g(u)=\frac{2 u^{2}}{5},
$$

and initial data which consist in the difference of indicator functions of 2 disks:

$$
u^{0}(x, y)=\chi\left(\sqrt{(x+0.5)^{2}+(y+0.5)^{2}}<0.4\right)-\chi\left(\sqrt{(x-0.5)^{2}+(y-0.5)^{2}}<0.4\right) .
$$

Obviously, in order to work with a Courant number greater than $\frac{1}{2}$ (and therefore to maximize accuracy by minimizing numerical dissipation), this test-case asks for the complete numerical flux functions involving nonlinear wave interactions, as explained in our §3.3. Numerical results in $t=2$ with $\Delta x=\Delta y=2^{-6}$ for a CFL number of 0.95 are displayed on Fig. 4.4: on the right graphic, one sees that the schemes differ from each other in the area where the solution develops "fingers", precisely on their boundaries, where delicate $2 \mathrm{D}$ effects play a role.
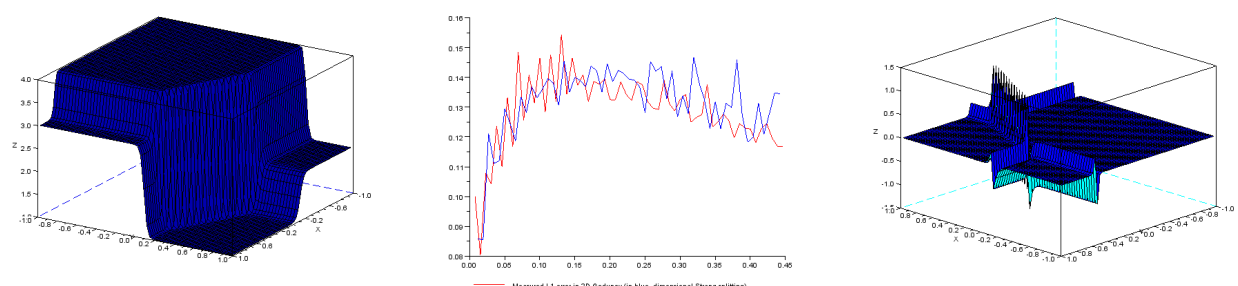

Fig. 4.5. Yoon-Hwang 4-shock benchmark (4.1) at time $t=0.45(C F L=0.985)$.

\subsection{D Riemann problems for homogeneous equations.}

- This "4-shocks" benchmark is taken from [53], §4.1. Its entropy solution is:

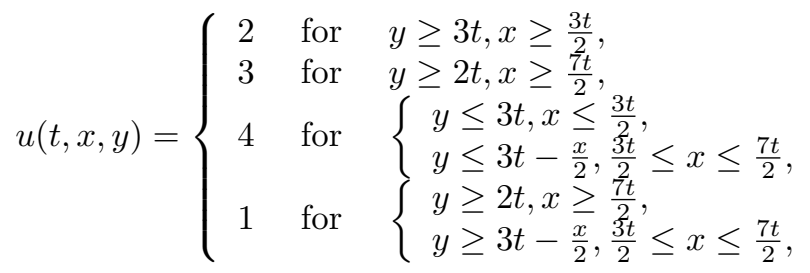

The Fig. 4.5 displays, on the left, the outcome of the 2D Godunov scheme for which the initial data was translated according to $x \rightarrow x+0.8, y \rightarrow y+0.8$, in 
order to track the scheme's behavior for longer time on the same computational domain $(-1,1) \times(-1,1)$. On the right, the pointwise difference with respect to the exact solution is shown at time $t=0.45$ and in the middle, the timeevolution of the $L^{1}$ error for both schemes, which are very similar with a small advantage going to the present $2 \mathrm{D}$ approach. The time-evolution in $L^{\infty}$ displays a similar behavior. As one may have expected, the pointwise errors on the discontinuities which are aligned with the computational grid are much smaller than the ones on the shock which is transversal.
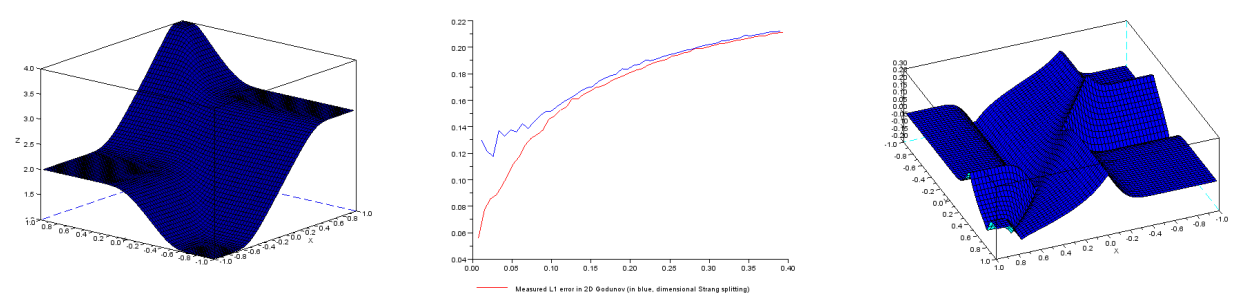

FIG. 4.6. Yoon-Hwang 4-rarefaction benchmark (4.2) at time $t=0.4(C F L=0.95)$.

- We now switch to another test-case proposed in [53], §4.5, namely the interaction of 4 rarefaction waves. Accordingly, its exact entropy solution is given by:

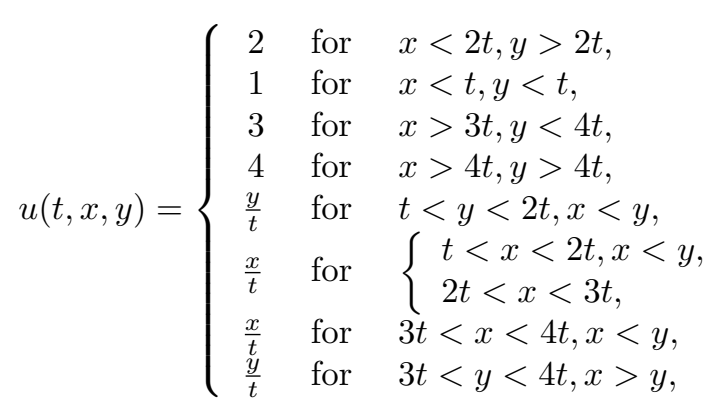

The presentation of numerical results in Fig. 4.6 exactly follows the one of Fig. 4.5, with the same $x, y$-translations: the main difference is that the timeevolution of the $L^{1}$ norm of both schemes' errors is smooth. Observe that it appears clearly as being of the order of $\sqrt{t}$ as stated in [42]. The outcome of the 2D Godunov scheme at time $t=0.4$ and its pointwise difference with respect to (4.2) appears on the left and right sides of Fig. 4.5, respectively.
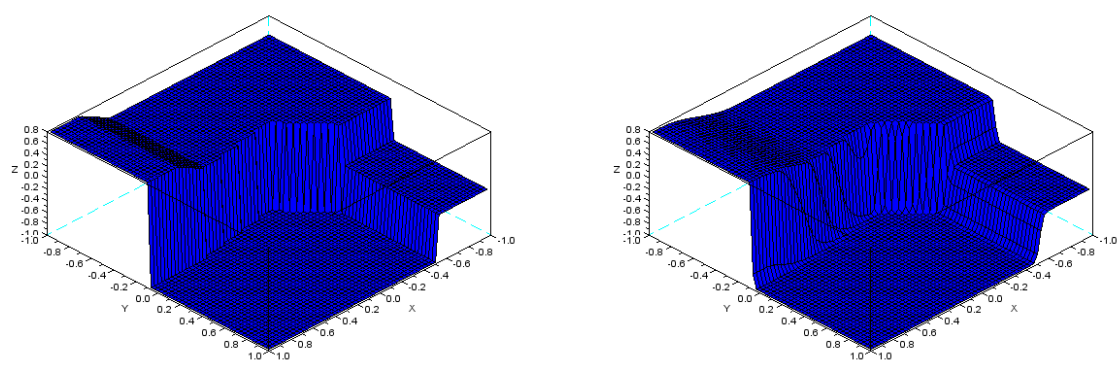

FIG. 4.7. Visualization of Jiang-Tadmor benchmark: exact (left), 2D-Godunov (right) at $t=0.9$. 
- We close this subsection with a more difficult benchmark, proposed in in [28] (page 1906) for very compressive situations. To treat it by means of the $2 \mathrm{D}$ Godunov scheme with a CFL number greater than $\frac{1}{2}$, it is necessary to include wave interactions (3.6) in the numerical flux functions. Its exact (and rather intricate) solution is given in the paper [22], on page 4258. A comparison between the exact entropy solution and the outcome of the 2D Godunov scheme with a Courant number of $0.8 \gg \frac{1}{2}$ at time $t=0.9$ is displayed on Fig. 4.7. In order to study the evolution in time of the pointwise discrepancies of both our 2D Godunov scheme and the more classical dimensional Strangsplitting scheme on this Riemann problem involving strong shock/rarefaction interactions, a time-evolution of the absolute differences between each scheme's numerical approximation and the available exact solution is shown in Fig. 4.8. On the left column of Fig. 4.8, the isolines of our 2D Godunov approximation
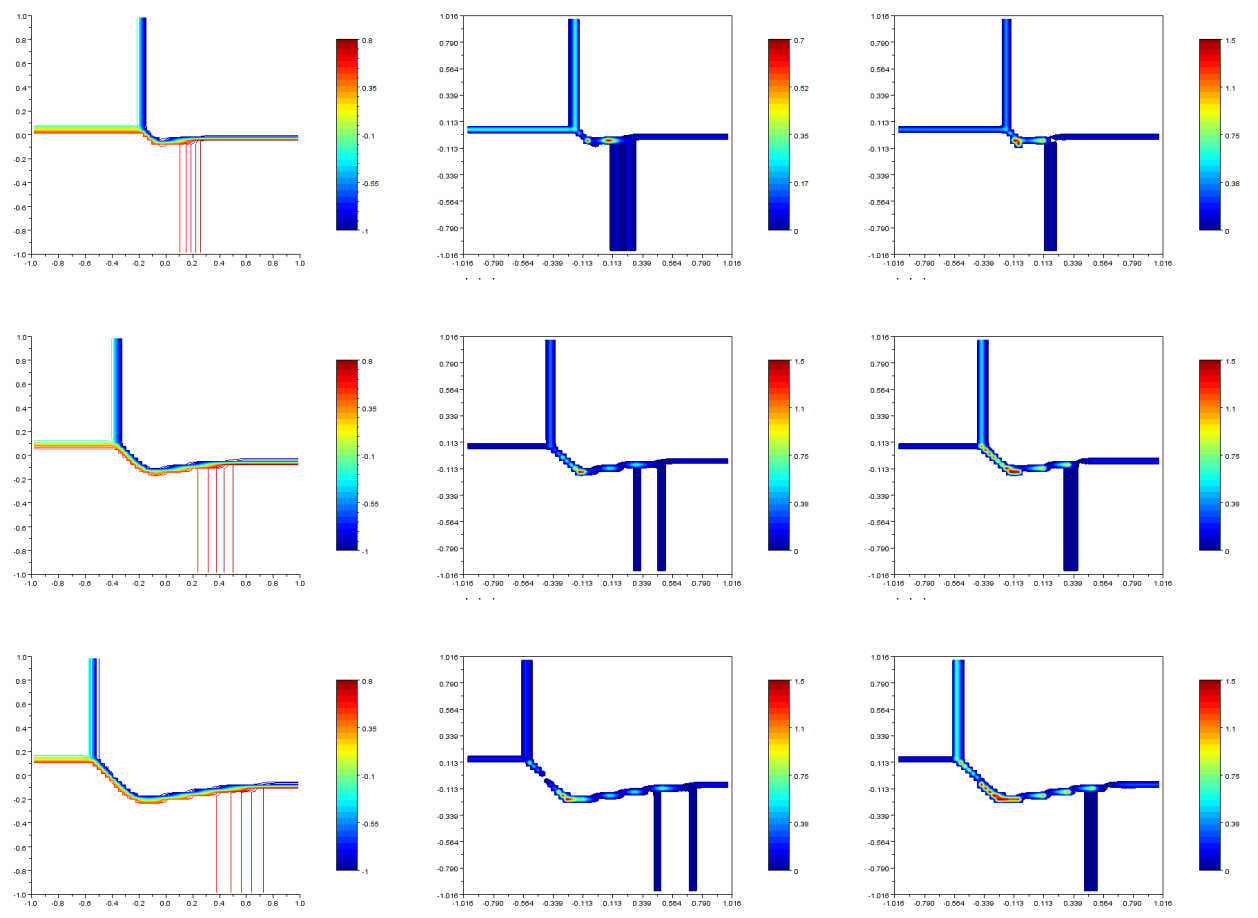

FIG. 4.8. Jiang-Tadmor compressive benchmark at times $t=0.3,0.6,0.9$ (top to bottom).

are displayed. The middle and right columns reveal the location of the pointwise errors (with respect to the exact solution) of our 2D Godunov scheme and the Strang-split scheme, respectively. It is very apparent that the main places where the 2D scheme over-performs correspond to the ones where interactions between different nonlinear waves occur, like for instance the strong diagonal shock propagating toward the $(-1,-1)$-corner and interacting with the small (vertical) rarefaction wave with positive velocity.

4.4. A simple case of inhomogeneous equation. Here the simple $2 \mathrm{D}$ test-case suggested in [20] (see pages 146/147) is reconsidered with the following equation,

$$
\partial_{t} u+\partial_{x} \frac{u^{2}}{2}+\partial_{y} \frac{u^{2}}{2}=u, \quad u(t=0, x, y)=\chi(x+0.8<0) \chi(y+0.8<0),
$$


in the computational domain $(x, y) \in(-1,1)^{2}$. Its exact entropy solution is simple:

$$
u(t, x, y)=\exp (t) \chi\left(x+0.8<\frac{1}{2}(\exp (t)-1)\right) \chi\left(y+0.8<\frac{1}{2}(\exp (t)-1)\right) .
$$

The time-step $\Delta t$ is chosen adaptively at each iteration with a fixed CFL number of
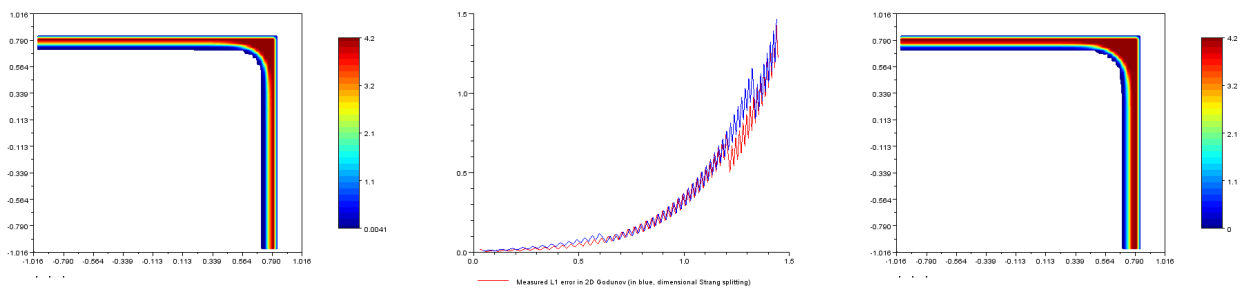

FIG. 4.9. Comparison between 2D-WB Godunov and dimensional-source Strang-split schemes.

0.95. Numerical results at time $t=1.45$ are given in Fig. 4.9 , where the present $2 \mathrm{D}$ WB scheme (2.15)-(2.17) is tested against the dimensional/source Strang-splitting scheme: pointwise errors with respect to the exact solutions are displayed on the left/right side, and the time-evolution of measured $L^{1}$ errors appear in the middle. This time-evolution reveal that the propagation of the 2 shocks is more accurate in the 2D Godunov scheme, because despite its oscillating nature, its $L^{1}$ error is always below the one of the splitting algorithm. However, even in the presence of a well-balanced treatment of the (very elementary) source term involving no explicit spatial dependence, the time-evolution of the measured $L^{1}$ error appears to be exponential in time: hence, the "one-dimensional miracle", that is, the linear in time growth of $L^{1}$ errors shown for well-balanced schemes in [3] doesn't seem to occur in 2D.

Following again [3] (see Fig.1 page 471), it may be interesting to simulate the same benchmark for equation (4.3), that is to say a rarefaction wave exponentially amplified. The corresponding initial data is now: $u(t=0, x, y)=\chi(x+0.8>0) \chi(y+0.8>0)$. Its exact entropy solution reads for any $t>0$ :

$$
u(t, x, y)=\left\{\begin{array}{lll}
\exp (t) & \text { for } \quad x>\exp (t)-1, y>\exp (t)-1 \\
\frac{x}{1-\exp (-t))} & \text { for } \quad 0<x \leq \exp (t)-1, x<y \\
\frac{y}{1-\exp (-t))} & \text { for } \quad 0<y<\exp (t)-1, x>y \\
0 & \text { for } \quad x<0, y<0
\end{array}\right.
$$

On Fig. 4.10, one can notice the same phenomenon than on Fig. 4.1, namely the
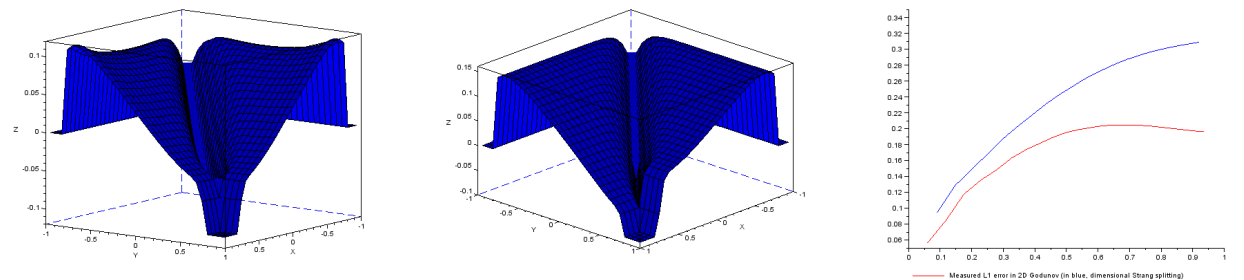

FIG. 4.10. Comparison between 2D-WB Godunov and dimensional-source Strang-split schemes.

asymmetrical character of the pointwise error generated by the Strang-split scheme (see the graphic in the middle). Oppositely, the pointwise error of the 2D WB scheme remains perfectly symmetrical with respect to the diagonal $x=y$. The time-evolution of the $L^{1}$ error for both scheme reveals the overall better quality of the numerical 
approximation obtained by means of (2.15)-(2.17). Lastly, since for $(4.3), k(x, y) \equiv 1$, one may think that the decomposition into an irrotational vector field $\left(K_{x}, K_{y}\right)=\nabla \omega$ shouldn't have noticeable effects on the 2D WB scheme's behavior. By setting up the trivial decomposition $K_{x}=K_{y} \equiv \frac{1}{2}$, one obtains an $L^{1}$ error roughly $10 \%$ bigger.

4.5. Three non-convex benchmarks. The convergence result stated in Theorem 1 doesn't obviously cover equations of the type (1.1)-(1.2) involving smooth, but non-convex fluxes. The whole process of constructing the $2 \mathrm{D}$ numerical fluxes may be tackled in its complexity, but one may also be tempted to extend "naively" the present $2 \mathrm{D}$ fluxes in the following manner (see [34], page 145):

$$
\begin{gathered}
F_{i-\frac{1}{2}, j}^{n}=\chi\left(u_{i-1, j}^{n} \leq u_{i, j}^{n}\right) \min _{u_{i-1, j}^{n} \leq u \leq u_{i, j}^{n}} f(u) \\
+\chi\left(u_{i-1, j}^{n}>u_{i, j}^{n}\right) \underset{u_{i, j}^{n} \leq u \leq u_{i-1, j}^{n}}{ } f(u) \\
+ \text { tangential terms. }
\end{gathered}
$$

The first non-convex benchmark we display hereafter deals with the so-called "Guckenheimer structure" [24, 54]: it involves a 3-state Riemann problem with initial data specified in $\S 3.2$ of [5] and fluxes $f(u)=\frac{u^{2}}{2}, g(u)=\frac{u^{3}}{3}$. Results coming from our 2D
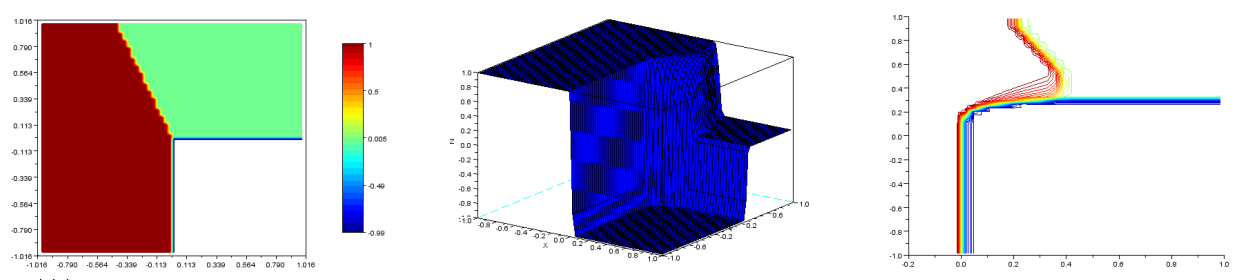

FiG. 4.11. Guckenheimer data (left) and emerging structure at time $t=0.95(C F L=0.7)$.

Godunov scheme are displayed on Fig. 4.11; the ones coming out of the dimensional Strang-splitting scheme are very similar. A more challenging non-convex benchmark was proposed by the authors of [31] and is referred to as the KPP rotating wave. One considers the equation (1.1)-(1.2) with periodic boundary conditions on the square $(-1,1)^{2}$ and the oscillating fluxes:

$$
f(u)=\sin u, \quad g(u)=\cos u, \quad u^{0}(x, y)=\frac{13 \pi}{4} \chi\left(x^{2}+y^{2}<1\right)+\frac{\pi}{4} .
$$

The outcome of our 2D Godunov scheme at time $t=1$ with a Courant number of $\frac{1}{2}$
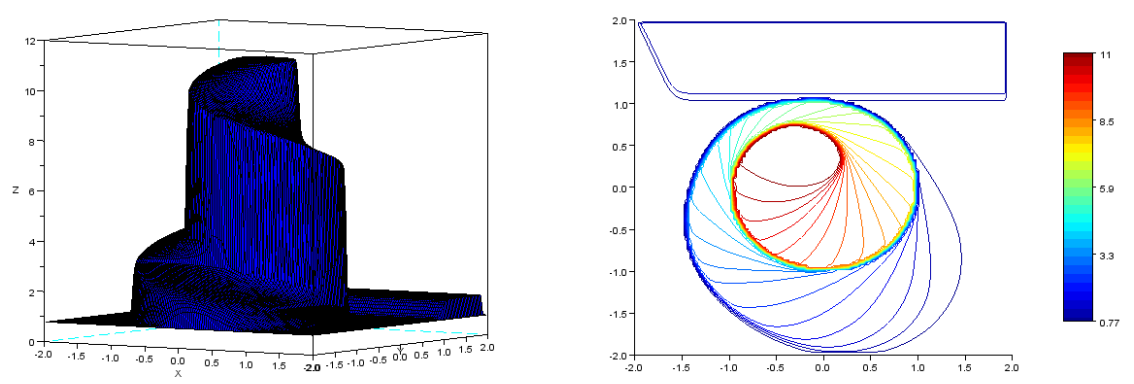

FIG. 4.12. KPP rotating pattern at time $t=1(C F L=0.5)$.

appears to be a "Babel tower" which is displayed on Fig. 4.12. Since all the derivations 
were made under the convexity assumption, such a result can be considered as being very satisfying. However, unphysical oscillations can develop in $u^{\Delta t}$ if CFL numbers close to 1 are prescribed. At last, following [25], one can consider a Gaussian initial data for a Buckley-Leverett equation with fluxes:

$$
f(u)=g(u)=\frac{1}{1+\alpha\left(\frac{1}{u}-1\right)^{2}}, \quad \alpha=2 .
$$

The results displayed on Fig. 4.13 at time $t=0.3$ were obtained with the same initial
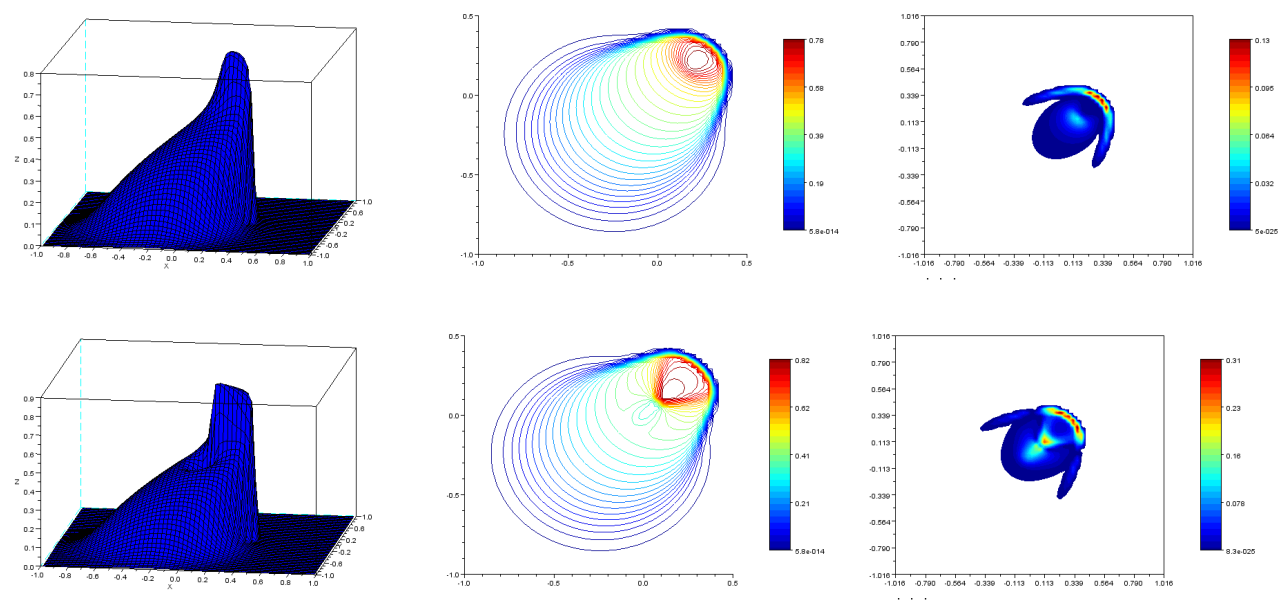

FIG. 4.13. Buckley-Leverett: 2D vs. Strang-split schemes, CFL=0.45 (top) 8 0.95 (bottom).

data as Fig. 4.3 and different CFL numbers. On the top line, one sees that the $2 \mathrm{D}$ Godunov scheme is very similar to the dimensional Strang-split scheme for a Courant number of 0.45 . However, the bottom line shows that, unfortunately, a loss of entropy consistency occurs for this equation when the Courant number becomes greater than $\frac{1}{2}$ : this means that the treatment of nonlinear wave interactions which is presented in $\S 3.3$ is not sufficient to keep monotonicity in this non-convex case.

5. Conclusion and outlook. It is shown that, based on early ideas proposed by Boukadida and LeRoux [6], a 2D Godunov scheme can be derived for scalar balance laws. Extensive numerical tests suggest that such derivations allow to get approximations where fine geometric features are better rendered. In particular, this algorithm competes with second order Strang-splitting while being of first order and monotone.

An important question is the ability of such a scheme to be extended, at a reasonable CPU cost, toward the case of more intricate hyperbolic systems of $2 \mathrm{D}$ balance laws. As a first step, it appears reasonable to derive genuinely bidimensional approaches for linearizations of such complex systems: this matches the ideas developed in $[8,17]$ (and in $[52,36]$ too, but following a slightly different method). However, progress still can hopefully be made in terms of derivation of $2 \mathrm{D}$ numerical fluxes.

\section{REFERENCES}

[1] R. Abgrall, Residual distribution schemes: current status and future trends, Computer \& Fluids, 35 (2006) 641-669.

[2] Saul Abarbanel, Adi Ditkowski, Bertil Gustafsson, On Error Bounds of Finite Difference Approximations to Partial Differential Equations-Temporal Behavior and Rate of Convergence $J$. Scient. Comput. 15 (2000), 79-116

[3] D. Amadori, L. Gosse, Transient $L^{1}$ error estimates for well-balanced schemes on non-resonant scalar balance laws, J. Differ. Equ. 255 (2013) 469-502. 
[4] D.N. Arnold, L.R. Scott, M. Vogelius, Regular inversion of the divergence operator with Dirichlet boundary conditions on a polygon, Ann. Scuola Norm. Sup. Pisa Cl. Sci. 15 (1988) 169-192.

[5] M. Ben-Artzi, J. Falcovitz, J. Li, Wave interactions and numerical approximation for twodimensional scalar conservation laws, Comput. Fluid Dynamics Journ. 14 (2006) 401-418

[6] T. Boukadida, A.-Y. LeRoux, A two-dimensional version of the Lax-Friedrichs scheme, Math. Comput. 63 (1994) 541-553

[7] M. Brio, A.R. Zakharian, G.M. Webb, Two-dimensional Riemann solver for Euler equations of gas dynamics, J. Comput. Phys. 167 (2001) 177-195

[8] Soumia Chaira, Sur la résolution numérique des systémes de la dynamique des gaz par des schémas multidimensionnels, Ph.D. Thesis, Ecole Normale Supérieure de Cachan (1995)

[9] G. Chen, D. Li, D. Tan, Structure of Riemann solutions for 2-dimensional scalar conservation laws, J. Differential Equations 127 (1996) 124-147.

[10] J. Cheng, C.-W. Shu, High order schemes for CFD: A review, Chinese Journal of Computational Physics, 26 (2009), 633-655.

[11] P. Colella, Multidimensional upwind methods for hyperbolic conservation laws, J. Comput. Phys., 87 (1990) 171-200

[12] R. M. Colombo, M. Garavello, M. Lcureux-Mercier, A Class of Non-Local Models for Pedestrian Traffic, C. R. Math. 349 769-772, 2011 and Math. Mod. \& Meth. in Applied Sci. 22 (2012)

[13] M. Crandall, A. Majda, Monotone Difference Approximations for Scalar Conservation Laws, Math. Comput. 34 (1980) 1-21

[14] S.F. Davis, A rotationally biased upwind difference scheme for the Euler equations, J. Comput. Phys. 56 (1984) 65-92

[15] C. De Lellis, F. Otto, M. Westdickenberg, Structure of Entropy Solutions for Multi-Dimensional Scalar Conservation Laws, Arch. Rat. Mech. Anal. 170 (2003), 137-184

[16] V. Elling, A possible counterexample to wellposedness of entropy solutions and to Godunov scheme convergence, Math. Comput. 75 (2006) 1721-1733

[17] H. Gilquin, J. Laurens, C. Rosier, Multi-dimensional Riemann problems for linear hyperbolic systems. RAIRO - Model. Math. \& Anal. Num., 30 (1996), 527-548

[18] J. Glimm et al., Front tracking and two dimensional Riemann problems, Adv. Appl. Math. 6 (1985), 259-290

[19] Jonathan B. Goodman and Randall J. LeVeque, On the Accuracy of Stable Schemes for 2D Scalar Conservation Laws, Math. Comput. 45 (1985) 15-21

[20] L. Gosse, A well-balanced flux-vector splitting scheme designed for hyperbolic systems of conservation laws with source terms, Comput. \& Math. Applic. 39 (2000) 135-159

[21] L. Gosse, Computing Qualitatively Correct Approximations of Balance Laws, Springer (2013) ISBN 978-88-470-2891-3

[22] J.-L. Guermond, R. Pasquetti, B. Popov, Entropy viscosity method for nonlinear conservation laws, J. Comput. Phys., 230 (2011) 4248-4267.

[23] V. Guinot, An approximate two-dimensional Riemann solver for hyperbolic systems of conservation laws, J. Comput. Phys. 205 (2005) 292-314

[24] J. Guckenheimer, Shocks and rarefactions in two space dimensions, Arch. Rational Mech. Anal. 59 (1975) 281-291

[25] H. Holden, K.H. Karlsen, K.-A. Lie, N.H. Risebro, Splitting Methods for Partial Differential Equations with Rough Solutions. Analysis and MATLAB programs. Series of Lectures in Mathematics. European Mathematical Society (EMS), Zürich, 2010

[26] L. Hsiao, Ch. Klingenberg, The structure of the solutions for the two dimensional Riemann problem, preprint SFB 123, University of Heidelberg (1986)

[27] E. Isaacson, B. Temple, Convergence of the $2 \times 2$ Godunov method for a general resonant nonlinear balance law, SIAM J. Appl. Math. 55 (1995) 625-640

[28] G.S. Jiamg, E. Tadmor, Non-oscillatory central schemes for multidimensional hyperbolic conservation laws, SIAM J. Scient. Comput. 19 (1998) 1892-1917.

[29] Ch. Klingenberg, S. Osher, Nonconvex Scalar Conservation Laws in One and Two Space Dimensions, in Nonlinear Hyperbolic Equations - Theory, Computation Methods, and Applications, Notes on Numerical Fluid Mechanics 24 (1989) 289-299

[30] S.N. Kružkov, First order quasilinear equations with several independent variables, Mat. Sbornik (N.S.), 81 (123):228255, 1970.

[31] A. Kurganov, E. Tadmor, Solution of Two-Dimensional Riemann Problems for Gas Dynamics without Riemann Problem Solvers, Numer. Meth. Partial Differ. Equ. 18 (2002) 548-608.

[32] P. Lax, X.D. Liu, Solution of twodimensional Riemann problem of gas dynamics by positive schemes. SIAM Sci Comp, (1998) 19319340

[33] Ph. LeFloch, A.E. Tzavaras, Representation of weak limits and definition of nonconservative products, SIAM J. Math. Anal. 30 (1999), 1309 - 1342.

[34] R. J. LeVeque, Numerical Methods for Conservation Laws, pp. 204-205, Lectures in Mathematics, ETH-Zurich Birkhauser-Verlag, Basel, 1990. ISBN 3-7643-2464-3

[35] R. J. LeVeque, High-resolution conservative algorithms for advection in incompressible flow, SIAM J. Numer. Anal. 33 (1996) 627-665.

[36] R. J. LeVeque, Wave Propagation Algorithms for Multidimensional Hyperbolic Systems, J. Comput. Phys. 131 (1997) 327-353. 
[37] Cai-Zhong Li, Tai-Ping Liu, Asymptotic states for hyperbolic conservation laws with a moving source, Adv. Applied Math. 4 (1983) 353-379

[38] J. Li, M. Lukacova, G. Warnecke, Evolution Galerkin schemes applied to two-dimensional Riemann problems for the wave equation system, DCDS-B 9 (2003) 559-576

[39] J. Li, W. Shen, T. Zhang, Y. Zheng, Two-Dimensional Riemann problems: from scalar conservation laws to compressible Euler equations, Acta Math. Sci. (English Ed.) 2009,29B(4) $777-802$.

[40] Jiequan Li, Tong Zhang, Shuli Yang, The Two-Dimensional Riemann Problem in Gas Dynamics, Pitman Monographs and Surveys in Pure and Applied Mathematics (1998) ISBN: 0582244080

[41] T.P. Liu, T. Yang, $L_{1}$ Stability for Systems of Hyperbolic Conservation Laws, Contemporary Mathematics 238, 1999 B 0-8218-1196-7-03547-7

[42] Brad Lucier, Error bounds for the methods of Glimm, Godunov, and LeVeque, SIAM J. Numer. Anal., 22 (1985), 1074-1081.

[43] M. Lukačova-Medviova, J. Saibertova, Finite volume schemes for multi-dimensional hyperbolic systems based on the use of bicharacteristics, Applic. Math. 51 (2006), 205-228

[44] S. Mishra, E. Tadmor (2010) Constraint preserving schemes using potential-based fluxes. I. Multidimensional transport equations Communications in Computational Physics 9(3) (2010), 688-710.

[45] D. Mitrea, Integral equation methods for Div-Curl problems for planar vector fields in nonsmooth domains, Differ. Integral Equ. 18 (2005) 1039-1054.

[46] J. Rauch, BV Estimates Fail for Most Quasilinear Hyperbolic Systems in Dimensions Greater Than One, Comm. Math. Phys. 106 (1986) 481-484.

[47] P.L. Roe, Discrete models for the numerical analysis of time-dependent multidimensional gas dynamics, J. Comput. Phys. 63458 (1986)

[48] P. L. Roe, D. Sidilkover, Optimum Positive Linear Schemes for Advection in Two and Three Dimensions, SIAM J. Numer. Anal. 291992

[49] C. L. Rumsey, B. Van Leer, P.L. Roe, A multi-dimensional flux function with applications to Euler and Navier-Stokes equations, J. Comput. Phys. 105, 306-323 (1993)

[50] E. Tadmor, M. Rascle, P. Bagnerini, Compensated compactness for $2 D$ conservation laws, J. Hyper. Differ. Equ. 2 (2005) 697-712

[51] D. Wagner, The Riemann problem in two space dimensions for a single conservation law, SIAM J. Math. Anal. 14 (1983), no. 3, 534-559.

[52] B. Wendroff, A Two-Dimensional HLLE Riemann Solver and Associated Godunov-Type Difference Scheme for Gas Dynamics, Comput. \& Math. Applic. 38 (1999) 175-185

[53] D. Yoon, W. Hwang, Two-dimensional Riemann problem for Burgers equation, Bull. Korean Math. Soc. 45 (2008), 191-205

[54] P. Zhang, T. Zhang, Generalized characteristic analysis and Guckenheimer structure, J. Differential Equations 152 (1999), no. 2, 409-430.

[55] Yuxi Zheng, Systems of Conservation Laws: Two-Dimensional Riemann Problems, Progress in Nonlinear Differential Equations and Their Applications 38 (Birkhauser Verlag) 